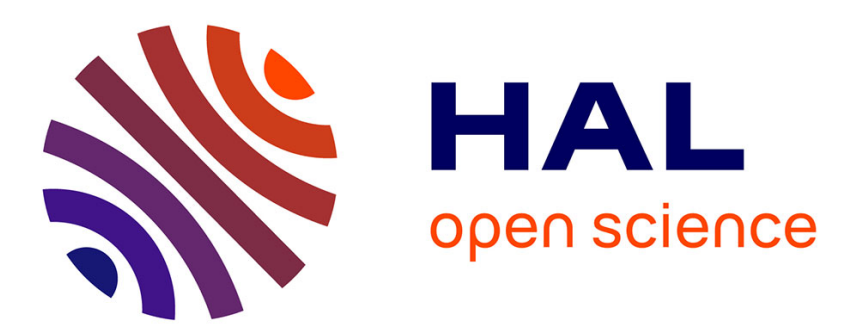

\title{
Multi-Area Throughput and Energy Optimization of UAV-aided Cellular Networks Powered by Solar Panels and Grid
}

Luca Chiaraviglio, Fabio d'Andreagiovanni, William Liu, Jairo Gutierrez, Nicola Blefari Melazzi, Kim-Kwang Raymond Choo, Mohamed-Slim Alouini

\section{To cite this version:}

Luca Chiaraviglio, Fabio d'Andreagiovanni, William Liu, Jairo Gutierrez, Nicola Blefari Melazzi, et al.. Multi-Area Throughput and Energy Optimization of UAV-aided Cellular Networks Powered by Solar Panels and Grid. IEEE Transactions on Mobile Computing, 2021, 20 (7), pp.2427-2444. 10.1109/TMC.2020.2980834 . hal-02503372

\section{HAL Id: hal-02503372 \\ https://hal.science/hal-02503372}

Submitted on 4 Jan 2021

HAL is a multi-disciplinary open access archive for the deposit and dissemination of scientific research documents, whether they are published or not. The documents may come from teaching and research institutions in France or abroad, or from public or private research centers.
L'archive ouverte pluridisciplinaire HAL, est destinée au dépôt et à la diffusion de documents scientifiques de niveau recherche, publiés ou non, émanant des établissements d'enseignement et de recherche français ou étrangers, des laboratoires publics ou privés. 


\title{
Multi-Area Throughput and Energy Optimization of UAV-aided Cellular Networks Powered by Solar Panels and Grid
}

\author{
Luca Chiaraviglio, ${ }^{(1,2)}$ Fabio D'Andreagiovanni, ${ }^{(3,4)}$ William Liu, ${ }^{5}$ Jairo A. Gutierrez, ${ }^{5}$ \\ Nicola Blefari-Melazzi, ${ }^{(1,2)}$ Kim-Kwang Raymond Choo, ${ }^{6}$ Mohamed-Slim Alouini ${ }^{7}$ \\ 1) University of Rome Tor Vergata, Italy, \{luca.chiaraviglio,blefari\}@uniroma2.it \\ 2) Consorzio Nazionale Interunivesitario per le Telecomunicazioni, Italy \\ 3) National Center for Scientific Research (CNRS), France \\ 4) Sorbonne Universités, Université de Technologie de Compiègne, CNRS, France, d.andreagiovanni@hds.utc.fr \\ 5) Auckland University of Technology, New Zealand, \{jairo.gutierrez, william.liu\}@ut.ac.nz \\ 6) The University of Texas at San Antonio, Texas, raymond.choo@fulbrightmail.org \\ 7) King Abdullah University of Science and Technology (KAUST), Kingdom of Saudi Arabia, slim.alouini@kaust.edu.sa
}

\begin{abstract}
Small Cells (SCs) mounted on top of Unmanned Aerial Vehicles (UAVs) can be used to boost the radio capacity in hotspot zones. However, UAV-SCs are subject to tight battery constraints, resulting in frequent recharges operated at the ground sites. To meet the UAV-SCs energy demanded to the ground sites, the operator leverages a set of Solar Panels (SPs) and grid connection. In this work, we demonstrate that both i) the level of throughput provided to a set of areas and ii) the amount of energy that is exchanged with the grid by the ground sites play a critical role in such UAV-aided cellular network. We then formulate the J-MATE model to jointly optimize the energy and throughput through revenue and cost components. In addition, we design the BBSR algorithm, which is able to retrieve a solution even for large problem instances. We evaluate J-MATE and BBSR over a realistic scenario composed of dozens of areas and multiple ground sites, showing that: i) both J-MATE and BBSR outperform previous approaches targeting either the throughput maximization or the energy minimization, and ii) the computation time and the memory occupation of BBSR are reduced up to five orders of magnitude compared to J-MATE.
\end{abstract}

Index Terms—cellular networks, throughput and energy management, UAV, renewable energy

\section{INTRODUCTION}

The potential to utilize Unammed Aerial Vehicles (UAVs) to carry Small Cell (SC) capabilities is a topic of ongoing interest in the research and practitioner community, as evidenced by the seminal works of [1], [2], [3] and the recent surveys/tutorials [4], [5]. In comparison to a traditional cellular network composed of fixed Base Stations (BSs), UAV-SCs provide: i) coverage flexibility [6], as it is possible to selectively choose the areas to be served by UAV-SCs over time and space, ii) performance increase [7], due to the Line Of Sight (LOS) and proximity conditions experienced on the radio link between the users and the serving UAV-SCs, and iii) CAPital EXpenditures (CAPEX) reduction [8], as the number of sites to host fixed BS capabilities is decreased. In this context, a viable option is to deploy a UAV-aided cellular network [9], in which a small number of Macro Cells (MCs) provide basic performance to users over the territory, while UAV-SCs are instead used to improve the capacity in selected areas.

Although the utilization of a UAV-aided cellular network is a promising approach for the deployment of future cellular networks [10], there are a number of technological challenges that need to be taken into account in real-world implementations of such architecture [5]. One key issue is the energy consumption of UAV-SCs [4]. Compared to a fixed BS, which is connected to the electricity grid, the battery capability of a UAV-SC is generally limited [11]. Consequently, the coverage of an area served by a UAV-SC has to be scheduled inside a mission [12] to always ensure an adequate battery level and avoid its discharge before the mission completion. In this context, each UAV-SC mission starts and ends at a set of ground sites, which provide recharging capabilities to the UAV-SCs. Since the cumulative energy required by the UAV-SCs that need to be recharged in a ground site may be significant [8], each ground site generally needs to consider (or attempts to leverage): i) the energy locally produced by a set Solar Panels (SPs) and ii) the connection to the electricity grid. In this way, the ground site can satisfy the UAV-SCs demand by buying energy from the grid when the energy produced by SPs is not sufficient and/or not available.

In such a scenario, it is clear that the energy-efficient management of UAV-SCs missions is a crucial aspect faced by the operator. From an OPerating EXpenses (OPEX) perspective, the operator should minimize the use of UAV-SCs, in order to: i) limit the number of UAV-SC recharges, and ii) maximize the amount of SP energy that is sold to the grid. However, this policy contradicts the need to provision the radio capacity to users, which targets the maximization of the UAV-SCs deployment to maximize the performance of the covered areas. In addition, the amount of throughput provided to the users may be linked to the revenue gained by the operator from users' subscription plan. This is especially true for data-hungry services like adaptive video streaming, Internet of Things (IoT) subscriptions, social 
TABLE 1

Positioning of this work w.r.t. the literature.

\begin{tabular}{|c|c|c|c|c|c|c|c|c|c|}
\hline Work & $\begin{array}{c}\text { Trajectory } \\
\text { Control }\end{array}$ & $\begin{array}{c}\text { Trajectory } \\
\text { Computation }\end{array}$ & Target & RRM & Fixed BSs & $\begin{array}{l}\text { Number } \\
\text { of UAVs }\end{array}$ & $\begin{array}{l}\text { UAV Energy } \\
\text { Constraints }\end{array}$ & $\begin{array}{l}\text { Site Energy } \\
\text { Constraints }\end{array}$ & Approach \\
\hline Mozaffari et al. [13] & - & - & Areas & - & - & Multiple & - & - & Opt. \\
\hline Sharma et al. [7] & - & - & Areas & - & Multiple & Multiple & - & - & Alg. \\
\hline Qureshi et al. [14] & - & - & Areas & - & - & Multiple & - & - & Model \\
\hline Mignardi et al. [15] & Yes & 2D-plane & Areas & - & Multiple & Multiple & - & - & Alg. \\
\hline Jeong et al. [16] & Yes & 2D-plane & Users & Yes (frames) & - & Single & Yes & - & Opt.,Alg. \\
\hline Verdone et al. [17] & Yes & 2D-plane & Areas & Yes (units) & Multiple & Single & - & - & Alg. \\
\hline Wu et al. [18] & Yes & 2D-plane & Users & - & - & Multiple & - & - & Opt.,Alg. \\
\hline Zhu et al. [19] & Yes & 2D-plane & Areas & - & - & Single & Yes & - & Opt.,Alg. \\
\hline Li et al. [20] & Yes & 3D-plane & Users & Yes (subcar.) & - & Single & - & - & Opt.,Alg. \\
\hline Mardani et al. [21] & Yes & 2D-plane & Areas & - & - & Single & Yes & - & Alg. \\
\hline Zeng et al. [22] & - & - & Users & - & Multiple & Multiple & - & - & Model \\
\hline Trotta et al. [23] & - & - & Areas & - & - & Multiple & Yes & Yes (Rec. actions) & Opt.,Alg. \\
\hline Wu et al. [24] & Yes & 2D-plane & Users & - & - & Multiple & Yes & - & Model \\
\hline Hua et al. [25] & Yes & 2D-plane & Users & Yes (BW) & Single & Single & Yes & - & Opt.,Alg. \\
\hline Sun et al. [26] & Yes & 3D-plane & Users & Yes (subcar.) & - & Single & Yes & - & Opt.,Alg. \\
\hline Chiaraviglio et al. [27] & Yes & MP graph & Areas & Yes (BW) & Single & Multiple & Yes & Yes (Single Site) & Opt. \\
\hline This work & Yes & MP graph & Areas & Yes (BW) & Multiple & Multiple & Yes & Yes (Multiple Sites) & Opt.,Alg. \\
\hline
\end{tabular}

media, which may be on a pay-as-you-go fee plan.

The optimization of the different costs/revenues terms derived from the management of the UAV-SCs is therefore a challenging aspect for an operator. In this context, a natural question is: Is it possible to jointly optimize the energy bought from the grid, the energy sold to the grid and the throughput provided by a UAV-aided cellular network architecture to maximize the global operator's revenue? The goal of our work is to answer this question, which, to the best of our knowledge, has received very little attention by the research community so far. Specifically, we develop a framework that allows the operator to control: i) the throughput provided over the territory by a set of MCs and a set of UAV-SCs, ii) the UAV-SCs missions over time and space, iii) the UAV-SC energy constraints and iv) the energy balance between the SPs production, the energy bought from the grid and the energy sold to the grid in the ground sites. Our contributions can be summarized as follows:

- we model the Radio Resource Management (RRM) operated by the MCs and the UAV-SCs, through the BandWidth (BW) that each BS distributes over the territory;

- we consider the BW redistribution operated over the territory in a scenario composed of multiple MCs and multiple UAV-SCs covering a set of areas;

- we model the UAV-SCs missions and energy levels over time and 3D-space domains through a graphbased approach;

- we introduce constraints to model the energy balance at the ground sites;

- we formalize an innovative optimization model, hereafter referred as J-MATE, to maximize the operator revenue;

- we design a new algorithm, hereafter referred as BBSR, targeting the solution of the problem in large instances, composed of dozens of areas and multiple ground sites;

- we consider a realistic scenario, located in Rotorua (New Zealand), which integrates real measurements and realistic parameters. To this aim, we also validate through a real testbed the UAV-SC energy consumption model defined in this work;

- we perform an in-depth analysis of the performance of J-MATE and BBSR compared to a set of other strategies used as reference;

- we consider a wide set of metrics to more accurately benchmark our proposed approach with the reference strategies.

Our results demonstrate J-MATE and BBSR are very effective in managing the revenues and costs components of the UAV-aided cellular architecture. Specifically, by leveraging a weight parameter assigned to the throughput, the operator can achieve: i) the maximization of the energy sold to the grid, ii) the maximization of the throughput provided to a set of areas, or iii) a balanced strategy between i) and ii). In addition, we also show that BBSR reduces both the computation time and the memory occupation up to five orders of magnitude compared to J-MATE. We believe that our outcomes may trigger future research in the field, which includes: i) the cooperation of multiple UAV-SCs to cover the same area and realize beyond 5G services; and ii) the design of machine learning-based algorithms to provide the capability to react in real time to unexpected events (e.g., bad weather conditions, UAV-SCs failures, user mobility).

The rest of the paper is organized as follows. Sec. 2 briefly reviews the related literature. Sec. 3 highlights the main features of the UAV-aided cellular architecture considered in this work. Sec. 4 and Sec. 5 present J-MATE and BBSR, respectively. The real-world scenario is shown in Sec. 6. Performance evaluation of the considered strategies is presented in Sec. 7. Finally, Sec. 8 concludes our work.

\section{Related Work}

Tab. 1 summarizes the positioning of this work compared to the literature, considering: i) the trajectory control of UAV-SCs (considered or not by the related work), ii) the computation of the trajectory (through a 2D plane, a 3D plane, or a multi-period (MP) graph), iii) the granularity of the targeted optimization (in terms of single user or whole areas), iv) the RRM policy implemented (none, frame-based, unit-based, subcarrier-based or BW-based), v) the number of fixed BSs assumed in the scenario (none, one single BS, or multiple BSs), vi) the considered number of UAV-SCs (single or multiple ), vii) the presence of constraints to control the UAV-SCs energy consumption, viii) the presence of constraints to control the site energy production/consumption, 
and ix) the pursued approach (model-based, optimizationbased, and/or algorithm-based).

Several considerations can be derived by observing in more detail Tab. 1. First of all, the literature on the topic may appear pretty vast at a first glance. However, many works introduce different simplifications, like the computation of the UAV-SCs trajectory in the $2 \mathrm{D}$ plane (i.e., not considering the 3D space), no RRM, no fixed BSs, one single UAV$\mathrm{SC}$, and no energy constraints for the UAV-SCs and/or the ground sites. Although we recognize the importance of such previous works, we point out that our goal is to consider a realistic UAV-aided cellular architecture, where: i) we control the UAV-SCs trajectories in the 3D space through an MP graph, ii) we consider the BW provided to each area as the main resource to be managed through the RRM, iii) we focus on a scenario where each area may be served by multiple fixed BSs or by one UAV-SC - we also provide evidence from real measurements that the condition in which multiple BSs serve the same area is common in currently deployed cellular networks (especially in zones located at the cell border), iv) we jointly control a set of multiple UAVSCs and their actions over time (e.g., covering, moving, recharging), v) we explicitly model the energy constraints of the UAVs and of the ground sites, vi) we tackle the problem by providing the J-MATE formulation and the BBSR algorithm.

Actually, the closest paper to this work is [27], where the authors have targeted the optimization of the area throughput and the grid-connected microgeneration in a UAV-based scenario. However, as outlined in Tab. 1, our work is radically different from [27] under the following aspects: i) we consider an architecture composed of multiple fixed BSs (and not a single one like in [27]) to better reflect the deployment of a realistic cellular network, ii) we assume multiple sites providing energy capabilities to the UAVs (and not a single site like in [27]) - in this way, each UAV is able to choose at which site to recharge, iii) we face the problem not only from the optimization perspective, but also by designing the BBSR algorithm, which is able to efficiently retrieve a solution in a limited amount of time and with limited memory resources. In addition, another key feature of this work (and not covered at all by previous ones) is the validation of the UAV-SC energy model (derived from [26]) through the measurements performed on a real UAV-based testbed.

Summarizing, we face a scenario where the UAV-SCs offer coverage capabilities to a set of areas. Each area may be served by a set of multiple fixed BSs or by a UAV-SC. We model the UAV-SC trajectories (and their actions) as an MP graph, in order to precisely control the UAV-SCs positions and actions in the 3D space. We consider a set of ground sites offering recharging capabilities to the UAV-SCs. We take into account the energy management of the UAV-SCs, by properly considering the impact of the different UAVSC actions on the battery level. In addition, we consider the ground site energy constraints in terms of: i) energy produced by SPs, ii) energy bought from the grid, iii) energy sold to the grid. Compared to previous work, we propose a new approach for a UAV-aided cellular network, which allows the operator to jointly control: i) the management of the radio resources (in terms of $\mathrm{BW}$ ) to provide the throughput over a set of areas, ii) the amount of energy exchanged with the grid by each ground site, iii) the actions, the trajectories and the battery levels of a set of UAV-SCs.

\section{ARCHITECTURE}

We describe our architecture through a set of main pillars, reported in the following subsections.

\subsection{UAV-SCs to improve the area throughput}

We exploit UAV-SCs to improve the throughput for a set of areas, where users and/or their activity tend to concentrate. Each UAV carries a Small Cell (SC), whose frequency band is separated from the one used by the fixed Macro Cells (MCs). Therefore, there is no interference between a UAV$\mathrm{SC}$ and a fixed MC. ${ }^{1}$ Focusing on the interference between UAV-SCs, we assume that each area is served by at most one UAV-SC. In addition, the positions of the areas and their coverage size, which are provided as input to our problem, are designed in a way to limit the interference between different UAV-SCs covering neighboring areas at the same time. In this way, when a UAV-SC covers an area, all the users in the area which were previously connected to the serving MC are handovered to the UAV-SC. We also assume that the handovers are performed in a way to limit glitches and performance losses to users. In addition, we ensure that a UAV-SC covers an area for a sufficiently long amount of time, i.e., in the order of minutes. In this way, we avoid frequent handovers forced to users between the UAV-SC and the $\mathrm{MC}(\mathrm{s})$, which would otherwise impact the presented results. In this scenario, the UAV-SC brings a throughput increase to the served area, due to the fact that: i) dedicated radio resources (in terms of $\mathrm{BW}$ ) are allocated, ii) better channel conditions are generally experienced compared to the MCs coverage, thanks to the fact that the UAV-SC is in close proximity (i.e., at most few hundreds meters) and in Line of Sight (LoS) with the users in the area.

\subsection{Bandwidth redistribution}

A key point targeted by this work is the control of the BW redistribution among the terrestrial MCs when the UAVSCs are exploited. To better understand this aspect, Fig. 1(a) reports a simple scenario composed of two MCs ("MC1" and "MC2"), one area exclusively served by MC1 ("Area 1 1"), one area exclusively served by MC2 ("Area 4"), two areas mutually served by the two MCs ("Area 2" and "Area $\left.3^{\prime \prime}\right)$, one extra zone for delivering BW resources of $\mathrm{MC} 1$, and one extra zone for delivering BW resources of MC2. For simplicity, BW resources are denoted in terms of elements (represented by cubes in the subfigure), which are managed by each MC. In this scenario, Area 2 and Area 3 contain BW resources from both MCs, due to the fact that these areas are located at the coverage border for both MCs. Hence, in order to adequately serve such areas, a large amount of

1. In this paper, we assume that the radio link between the UAV$\mathrm{SC}$ and the ground site, which provides connectivity to the rest of the Internet, does not use the same portion of BW of the MCs or the UAVSCs. This link may be realized considering other radio technologies, such micro-wave links or Free Space Optical (FSO) links. We leave the investigation of this aspect as future work. 


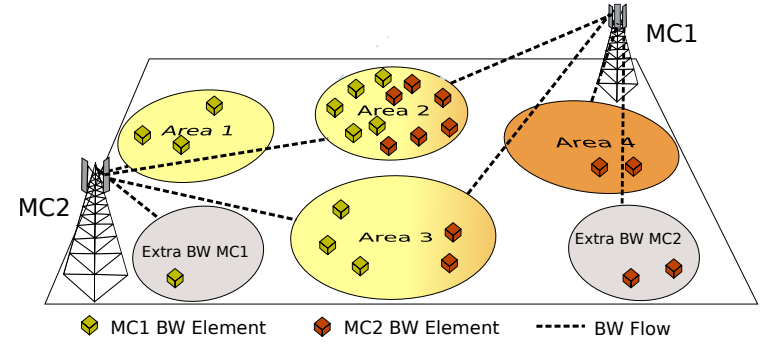

(a) All areas covered by the MCs.

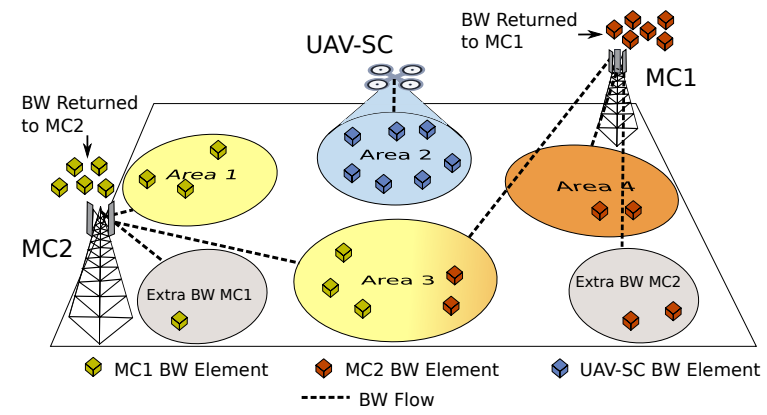

(b) Area 2 covered by a UAV-SC: BW release to the MCs

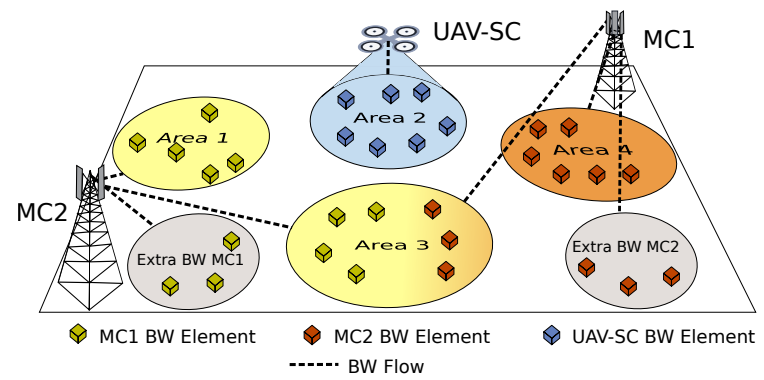

(c) BW reallocation by MCs

Fig. 1. MCs BW management: the BW resources assigned by MCs to Area 2 (subfigure a) are released to the MCs when Area 2 is covered by a UAV-SC (subfigure b) and they are then reallocated among the other areas not covered by UAV-SCs (subfigure c).

BW resources of the MCs is exploited. ${ }^{2}$ Now, let us suppose that Area 2 is served by a UAV-SC (Fig. 1(b)). When the SC on board the UAV starts covering the area, all the BW resources that where previously assigned to the area are released to the MCs. This pool of BW resources is then reassigned by the MCs (Fig. 1(c) ) to improve the throughput of the other areas not covered by UAV-SCs (see e.g., Area 4, which passes from 2 radio resources in Fig. 1(a) to 6 radio resources in Fig. 1(c)). In this way, the exploitation of UAV$\mathrm{SC}$ allows the $\mathrm{BW}$ release to the $\mathrm{MC}(\mathrm{s})$ previously serving the areas, and therefore a better throughput also to the areas not served by any UAV-SC. This is a fundamental aspect (not deeply covered by the UAV-related literature so far), taken into account by our work. Without the BW redistribution, the maximization of the UAV-SC throughput would result into the maximization of the number of areas covered by UAV-SCs over time. However, the BW redistribution feature makes this objective not trivial. In fact, the reallocation of BW may bring, for a specific area not covered by any

2. This assumption is reasonable, since users at the cell edge experience bad channel conditions. Therefore, in order to guarantee a given throughput, the MC has to consume a large number of BW resources for such users.

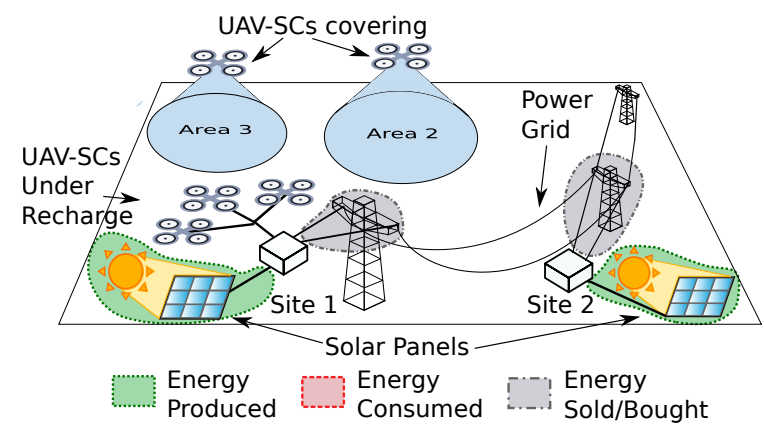

Fig. 2. Energy management at the ground sites. Each site balances the energy produced by the SPs, the energy consumed by the UAV-SCs under recharge (if any) and the energy exchanged with the grid.

UAV-SC, a notable BW improvement (and consequently a throughput increase). Our work properly considers also this feature.

\subsection{Energy management}

A third aspect addressed by this work is the management of the different energy components available in the set of ground sites, as shown in Fig. 2. More specifically, each ground site hosts charging stations on which the UAVSCs must land to recharge. To this aim, the ground site balances the energy required to recharge the UAV-SCs with the energy produced by the SPs and the energy bought or sold to the grid (see e.g., Site 1 in the figure). Clearly, from an energy cost point of view, the recharge of the UAV-SCs should be minimized, in order to allow the selling of the energy produced by the SPs directly to the grid (see e.g., Site 2 in the figure).

\subsection{UAV-SC mission scheduling}

Finally, we consider the modelling of the UAV-SC missions over time and space. We assume that the time is discretized in Time Slots (TSs). In each TS, each UAV-SC performs one of the following actions: staying parked at a ground site and not consuming any energy (STAY action), recharging at a ground site (REC), moving from a site to an area, or from an area to a site (MOV action), or covering an area (COV action). Fig. 3 reports a representative example showing a set of UAV-SCs, each of them performing a specific action. In this work, we precisely model the battery level of each UAV-SC, as a consequence of a given action set to a UAV-SC in a given location and at a given TS. This is also another fundamental aspect covered by this work, since the UAVs are subject to tight battery constraints [5]. More in depth, when a UAV-SC performs a REC action, its battery level is increased (see e.g., UAV-SC 2 in the figure). On the other hand, when the UAV-SC performs a MOV or a COV action, its battery level decreases. The amount of energy consumed by the MOV action depends on several parameters, like the flight path between the area and the site, the UAV-SC speed, the UAV-SC aerodynamic features and the cruise altitude, all of them taken into account by this work through a realistic energy consumption model. In addition, we assume that, when the UAV-SC performs a COV action, the SC functionalities are activated and the UAV-SC starts hovering over the area. This action is also integrated in our energy consumption model. 


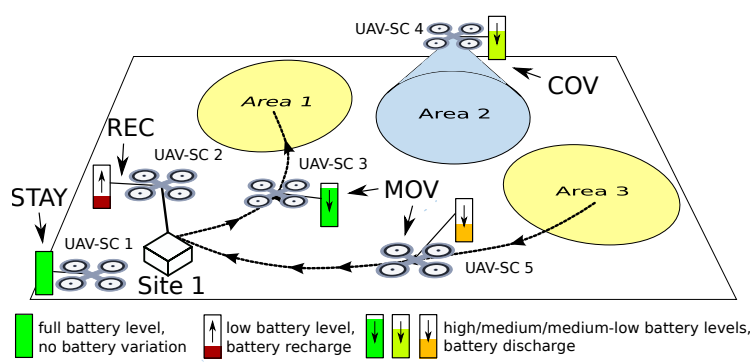

Fig. 3. UAV-SCs actions and battery levels in a sample scenario.

\section{J-MATE FORMULATION}

We formulate J-MATE in the following subsections: i) input sets and graph notation, ii) feasibility constraints, iii) objective function and complete model. The main notation introduced throughout the text is also summarized in Tab. 2.

\subsection{Input Sets and Graph Notation}

Let us denote with $A, S$ and $U$ the set of areas, the set of ground sites, and the set of UAV-SCs, respectively. Without loss of generality, we assume that each site $s \in S$ hosts MC functionalities. We denote with $P$ the set of places, defined as $P=A \cup S$. The set of Time Slots (TSs) is denoted as $T$. Moreover, we denote as $G(N, L)$ the multi-period graph, used to model the UAV-SCs missions over space and time. The set of nodes $N$ of the multi-period graph is defined as the union of the pairs $(p, t) \quad \forall p \in P, t \in T$, and two fictitious nodes $\Omega$ and $\Phi$, used to inject and collect tokens in $G$. $L$ denotes the set of links of $G$, which is defined as the union of different subsets, used to model the UAV-SCs actions. Specifically, we consider movement $\operatorname{arcs}\left(L^{\mathrm{MOV}}\right)$, coverage $\operatorname{arcs}\left(L^{\mathrm{COV}}\right)$, staying $\operatorname{arcs}\left(L^{\mathrm{STAY}}\right)$ and recharging $\operatorname{arcs}\left(L^{\mathrm{REC}}\right)$ to model the MOV, COV, STAY and REC actions, respectively. Each of the aforementioned arcs is defined between node $\left(p_{1}, t-1\right)$ and $\left(p_{2}, t\right)$, where $p_{1}, p_{2} \in P$ and $t \in T$. In other words, we consider arc connections on the graph between consecutive TSs. Clearly, it holds that: i) $p_{1} \neq p_{2}$ for $L^{\mathrm{MOV}}$, ii) $p_{1}=p_{2} \in A$ for $L^{\mathrm{COV}}$, iii) $p_{1}=p_{2} \in S$ for $L^{\mathrm{STAY}}$ and $L^{\mathrm{REC}}$. In addition, $L$ includes the fictitious $\operatorname{arcs} L^{\Omega}$ and $L^{\Phi}$, which are used to move the tokens from $\Omega$ and to $\Phi$, respectively. An arc $l \in L^{\Omega}$ is set between $\Omega$ and $\left(p, t_{\mathrm{START}}\right)$, where $p \in P$ and $t_{\mathrm{START}} \in T$ is the initial TS. On the other hand, an arc $l \in L^{\Phi}$ is set between $\left(p, t_{\mathrm{END}}\right)$ and $\Phi$, where $p \in P$ and $t_{\mathrm{END}} \in T$ is the last TS. Eventually, we denote the head $h(l)$ of arc $l \in L$ defined between nodes $\left(p_{1}, t_{1}\right)$ and $\left(p_{2}, t_{2}\right)$ as $h(l)=\left(p_{2}, t_{2}\right)$. In a similar way, we define the tail $t(l)$ of arc $l \in L$ as $t(l)=\left(p_{2}, t_{2}\right)$.

\subsection{Feasibility Constraints}

Flow Constraints. We initially introduce the binary flow variables $m_{(l, u)}$, which take value 1 if UAV-SC $u \in U$ activates link $l \in L, 0$ otherwise. We then impose the flow conservation over the multi-period graph with the following constraint:

$$
\sum_{\substack{l \in L: \\ t(l)=(p, t-1)}} m_{(l, u)}-\sum_{\substack{l \in L: \\ h(l)=(p, t)}} m_{(l, u)}=0
$$

TABLE 2

Main Notation.

\begin{tabular}{|c|c|c|}
\hline & Symbol & Description \\
\hline \multirow{15}{*}{ 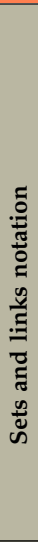 } & $A$ & Set of areas \\
\hline & $\begin{array}{l}P \\
P\end{array}$ & Set of places $(P=A \cup S)$ \\
\hline & $U$ & Set of UAV-SCs \\
\hline & $T$ & Set of Time Slots (TSs) \\
\hline & $\Omega, \Phi$ & Source and sink nodes \\
\hline & $\stackrel{N}{N}$ & Set of nodes $(N=(P \times T) \cup\{\Omega\} \cup\{\Phi\})$ \\
\hline & $L^{\text {STAY }}$ & Set of movement (MOV) links \\
\hline & $L^{\text {SIAY }}$ & Set of staying (STAY) links \\
\hline & $L^{\mathrm{COV}}$ & Set of coverage (COV) links \\
\hline & $L^{\mathrm{REC}}$ & Set of recharge (REC) links \\
\hline & $L^{\Omega}$ & Set of links from $\Omega$ node \\
\hline & $\begin{array}{c}L^{\Phi} \\
L\end{array}$ & $\begin{array}{l}\text { Set of links to } \Phi \text { node } \\
\text { Set of links }\left(L=L^{\mathrm{MOV}} \cup L^{\mathrm{STAY}} \cup L^{\mathrm{COV}} \cup L^{\mathrm{REC}} \cup L^{\Omega} \cup\right. \\
\left.L^{\Phi}\right)\end{array}$ \\
\hline & $G(N, L)$ & Multi-Period (MP) graph \\
\hline & $h(l)$ & Head node of link $l \in L$ \\
\hline & $t(l)$ & Tail node of link $l \in L$ \\
\hline \multirow{14}{*}{ 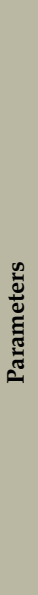 } & $E_{(s, t)}^{\mathrm{PROD}}$ & Energy produced by site $s$ at TS $t \in T$ \\
\hline & $E_{l}$ & Energy associated with link $l \in L$ \\
\hline & $W_{s}^{\mathrm{TOT}}$ & Total BW of MC installed in site $s \in S$ \\
\hline & $W_{(a, s)}^{\mathrm{BASE}}$ & $\begin{array}{l}\text { Baseline BW provided by } \mathrm{MC} \text { in site } s \in S \text { to area } \\
a \in A\end{array}$ \\
\hline & $W_{a}^{\mathrm{UAV}-\mathrm{SC}}$ & BW provided by a UAV-SC to area $a \in A$ \\
\hline & $B^{\mathrm{MIN}}$ & Minimum UAV-SC battery level \\
\hline & $B^{\mathrm{MAX}}$ & Maximum UAV-SC battery level \\
\hline & $O$ & Protocol overhead $(O \in(0,1])$ \\
\hline & $D_{(\mathrm{a}, \mathrm{s})}$ & $\begin{array}{l}\text { Coverage matrix ( } 1 \text { if area } a \in A \text { is covered by site } \\
s \in S, 0 \text { otherwise) }\end{array}$ \\
\hline & $F_{(a, s)}^{\mathrm{MC}}$ & $\begin{array}{l}\text { Spectral efficiency of area } a \in A \text { when served by MC } \\
\text { in site } s \in S\end{array}$ \\
\hline & $F_{a}^{\mathrm{UAV}-\mathrm{SC}}$ & $\begin{array}{l}\text { Spectral efficiency of area } a \in A \text { when served by a } \\
\text { UAV-SC }\end{array}$ \\
\hline & $R^{\text {RATE }}$ & Revenue for throughput \\
\hline & $R_{t}^{\mathrm{SELL}}$ & Revenue for selling energy at TS $t \in T$ \\
\hline & $C_{t}^{\mathrm{BUY}}$ & Cost for buying energy at TS $t \in T$ \\
\hline \multirow{10}{*}{ 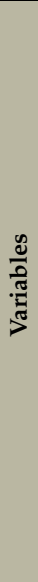 } & $m_{(l, u)}$ & $\begin{array}{l}\text { Binary flow variable ( } 1 \text { if UAV-SC } u \in U \text { activates link } \\
l \in L, 0 \text { otherwise) }\end{array}$ \\
\hline & $w_{(l, u)}$ & $\begin{array}{l}\text { Fractional energy variable of UAV-SC } u \in U \text { over link } \\
l \in L\left(0 \leq w_{(l, u)} \leq 1\right)\end{array}$ \\
\hline & $c_{(a, t)}$ & $\begin{array}{l}\text { Binary coverage variable ( } 1 \text { if area } a \in A \text { is covered by } \\
\text { one single UAV-SC at TS } t \in T, 0 \text { otherwise) }\end{array}$ \\
\hline & $b_{(u, t)}$ & $\begin{array}{l}\text { Battery level variable of UAV-SC } u \in U \text { in TS } t \in T \\
\left(B^{\mathrm{MIN}} \leq b_{(u, t)} \leq B^{\mathrm{MAX}}\right)\end{array}$ \\
\hline & $e_{(s, t)}^{\mathrm{BUY}}$ & Energy bought by site $s \in S$ at TS $t \in T\left(e_{(s, t)}^{\mathrm{BUY}} \geq 0\right)$ \\
\hline & $e_{(s, t)}^{\mathrm{SELL}}$ & Energy sold by site $s \in S$ at TS $t \in T\left(e_{(s, t)}^{\mathrm{SELL}} \geq 0\right)$ \\
\hline & $g_{(a, s)}^{t}$ & $\begin{array}{l}\text { Fraction of BW released to MC in site } s \in S \text { by area } \\
a \in A \text { when covered by a UAV-SC at TS } t \in T(0 \leq \\
\left.g_{(a, s)}^{t} \leq 1\right)\end{array}$ \\
\hline & $g_{(s, a)}^{t}$ & $\begin{array}{l}\text { Fraction of additional BW assigned by MC in site } s \in \\
S \text { to area } a \in A \text { at TS } t \in T\left(0 \leq g_{(s, a)}^{t} \leq 1\right)\end{array}$ \\
\hline & $\gamma_{s}^{t}$ & $\begin{array}{l}\text { Fraction of additional BW available at MC in site } s \in S \\
\text { at TS } t \in T \text { and not assigned to any area }\left(0 \leq \gamma_{s}^{t} \leq 1\right)\end{array}$ \\
\hline & $r_{(a, t)}$ & $\begin{array}{l}\text { Downlink throughput of area } a \in A \text { at TS } t \in T \\
\left(r_{(a, t)} \geq 0\right)\end{array}$ \\
\hline
\end{tabular}

In addition, node $\Omega$ is used to inject in the graph a single token for each UAV-SC:

$$
\sum_{\substack{l \in L: \\ t(l)=\Omega}} m_{(l, u)}=1 \quad \forall l \in L^{\Omega}, u \in U
$$

Moreover, node $\Phi$ captures the injected token for each UAV-SC through the following constraint:

$$
\sum_{\substack{l \in L: \\ h(l)=\Phi}} m_{(l, u)}=1 \quad \forall l \in L^{\Phi}, u \in U
$$

By including constraints (1), (2), (3), we impose that: i) each UAV-SC may be utilized starting from the initial TS up 
to the final one, and ii) the mission consistency is satisfied (i.e., the UAV-SCs always perform feasible missions).

Coverage Constraints. We then introduce the binary variable $c_{(a, t)}$, which takes 1 if area $a \in A$ is covered by one UAV-SC at TS $t \in T, 0$ otherwise. The value of $c_{(a, t)}$ is set through the following equation:

$$
\sum_{u \in U} \sum_{l \in L^{\operatorname{cov}}: h(l)=(a, t)} m_{(l, u)}=c_{(a, t)} \quad \forall a \in A, t \in T: t \geq 1
$$

Note that the previous constraint imposes that area $a$ is either covered by one single UAV-SC or not covered by any UAV-SC at TS $t$.

Energy Constraints. We introduce the continuous variable $w_{(l, u)} \in[0,1]$ associated with link $l \in L^{\mathrm{REC}}$ and UAVSC $u \in U$, in order to allow a partial recharge of UAV-SC battery (i.e., even lower than $E_{l}$ ). In this way, the energy requested by UAV-SC $u$ to recharge on link $l \in L^{\mathrm{REC}}$ is $E_{l} \cdot w_{(l, u)}$.

We then impose that $w_{(l, u)}$ can be strictly positive iff the corresponding flow variable is activated:

$$
w_{(l, u)} \leq m_{(l, u)} \quad \forall l \in L^{\mathrm{REC}}, u \in U
$$

In the following, we consider the energy balance implemented in the ground site. To this aim, let us denote with $E_{(s, t)}^{\mathrm{PROD}}$ the energy produced by SPs installed at site $s \in S$ during TS $t \in T . E_{(s, t)}^{\mathrm{PROD}}$ is an input parameter to our problem. We then introduce the continuous variables $e_{(s, t)}^{\mathrm{BUY}}$ and $e_{(s, t)}^{\mathrm{SELL}}$, which are used to denote the amount of energy that is bought from the grid or that is sold to the grid by site $s$ at TS $t$, respectively. Each site $s$ has to balance the energy requested by the UAV-SCs under recharge, the energy produced by SPs, and the energy exchanged with the grid. More formally, we have:

$$
\begin{array}{r}
\sum_{u \in U} E_{l} \cdot w_{(l, u)}-e_{(s, t)}^{\mathrm{BUY}}+e_{(s, t)}^{\mathrm{SELL}}=E_{(s, t)}^{\mathrm{PROD}} \\
\forall l \in L^{\mathrm{REC}}: h(l)=(s, t) \wedge t(l)=(s, t-1), s \in S, t \in T
\end{array}
$$

We then introduce the $w_{(l, u)}$ variables also for the MOV and COV link types, to make our notation homogeneous. Since fractional values of $E_{l}$ are not allowed for $L^{\mathrm{COV}}$ and $L^{\mathrm{MOV}}$ (i.e., the energy consumed by the UAV-SC is equal to $\left.E_{l}\right)$, we impose the following constraint:

$$
w_{(l, u)}=m_{(l, u)} \quad \forall l \in L^{\mathrm{COV}} \cup L^{\mathrm{MOV}}, u \in U
$$

Moreover, we model the UAV-SC battery level. We initially introduce the continuous variable $b_{(u, t)}$ to store the battery level of UAV-SC $u \in U$ at TS $t \in T$. We then compute the UAV-SC battery level as:

$$
b_{(u, t)}=b_{(u, t-1)}+\sum_{\substack{l \in L^{\mathrm{REC}} \cup L^{\mathrm{MOV}} \cup L^{\mathrm{COV}}: \\ t(l)=(*, t-1) \\ h(l)=(*, t)}} E_{l} \cdot w_{(l, u)}
$$

$\forall u \in U, t \in T$

We remind that the sign of $E_{l}$ values is positive for $l \in L^{\mathrm{REC}}$, and negative for $l \in L^{\mathrm{MOV}}$ and $l \in L^{\mathrm{COV}}$. In other words: a recharge action always increases the battery level, while a coverage or a movement action always decreases the battery level.
Finally, we introduce the input parameters $B^{\mathrm{MAX}}$ and $B^{\mathrm{MIN}}$ to denote the maximum and the minimum UAV-SC battery capacity, respectively. We then ensure that the UAVSC battery level is within these bounds:

$$
B^{\mathrm{MIN}} \leq b_{(u, t)} \leq B^{\mathrm{MAX}} \quad \forall u \in U, t \in T
$$

BW Redistribution Constraints. We initially model the amount of BW released to MC located at site $s \in S$ when a UAV-SC covers an area $a \in A$ at TS $t \in T$. To this aim, we introduce the continuous variable $g_{(a, s)}^{t}$ to store the fraction of BW released to MC in site $s$ when a UAVSC covers an area $a$ at TS $t$. In addition, let us denote with $W_{s}^{\mathrm{TOT}}$ the total BW used by MC in site $s$ and with $W_{(a, s)}^{\mathrm{BASE}}$ the amount of baseline BW assigned to area $a$ by MC in site $s$, respectively. In addition, let us denote with $D_{(a, s)}$ a binary matrix taking value 1 if area $a$ can receive $\mathrm{BW}$ from MC in site $s, 0$ otherwise. Clearly, both $W_{s}^{\mathrm{TOT}}, W_{(a, s)}^{\mathrm{BASE}}$ and $D_{(a, s)}$ are input parameters to our problem. The amount of BW released by area $a$ to MC in site $s$ at TS $t$ is then defined as:

$$
g_{(a, s)}^{t}=\frac{W_{(a, s)}^{\mathrm{BASE}}}{W_{s}^{\mathrm{TOT}}} \cdot D_{(a, s)} \cdot c_{(a, t)} \quad \forall a \in A, s \in S, t \in T
$$

We then consider the amount of BW that is redistributed to areas not covered by any UAV-SC. We introduce the continuous variable $g_{(s, a)}^{t}$ (having an inverse order of the indexes w.r.t. $g_{(a, s)}^{t}$, which denotes the fraction of additional BW that is assigned to area $a$ by MC in site $s$. Clearly, $g_{(s, a)}^{t}$ is equal to 0 if one of the following conditions hold: $\mathrm{i}$ ) the area $a$ is currently covered by a UAV-SC (and hence no BW from any MC should be assigned to the area) or ii) the area $a$ can not be covered by site $s$ (e.g., due to the fact that it is outside the coverage of $s$ ). Both the conditions are expressed through the following constraint:

$$
g_{(s, a)}^{t} \leq\left(1-c_{(a, t)}\right) \cdot D_{(a, s)} \quad \forall a \in A, s \in S, t \in T
$$

We then consider the management of released and assigned BW at each MC. To model this aspect, we impose a flow conservation between the variables $g_{(a, s)}^{t}$ and $g_{(s, a)}^{t}$. In addition, we introduce the continuous variable $\gamma_{s}^{t}$ to store the surplus of BW that is released from (some) areas and not used to cover other areas. We recall that this surplus of BW may be used by the MC for other needs, e.g., to serve the users under mobility or other zones that are not covered at all by UAV-SCs. More formally, we have:

$$
\sum_{a \in A} g_{(a, s)}^{t}=\sum_{a \in A} g_{(s, a)}^{t}+\gamma_{s}^{t} \quad \forall s \in S, t \in T
$$

Fig. 4 reports a representative example showing how the variables $g_{(a, s)}^{t}, g_{(s, a)}^{t}$ and $\gamma_{s}^{t}$ are governed by the constraints (10), (11), (12), the coverage variables $c_{(a, t)}$ and the input matrix $D_{(a, s)}$. We consider a toy-case scenario composed of two MCs (installed in $s_{1}$ and in $s_{2}$ ) and three areas $a_{1}$, $a_{2}$ and $a_{3}$. More in depth, $a_{1}$ is served by MC in $s_{1}, a_{2}$ is served by a UAV-SC, and $a_{3}$ is served by MC in $s_{2}$. For simplicity, we report also two extra zones, used to assign extra BW apart from the one delivered to $a_{1}, a_{2}$ and $a_{3}$. In this scenario, it holds that: $c_{\left(a_{1}, t\right)}=0, c_{\left(a_{2}, t\right)}=1$, $c_{\left(a_{3}, t\right)}=0$. Moreover, let us assume the following input matrix: $D_{\left(a_{1}, s_{1}\right)}=1, D_{\left(a_{1}, s_{2}\right)}=0, D_{\left(a_{2}, s_{1}\right)}=1, D_{\left(a_{2}, s_{2}\right)}=1$, 


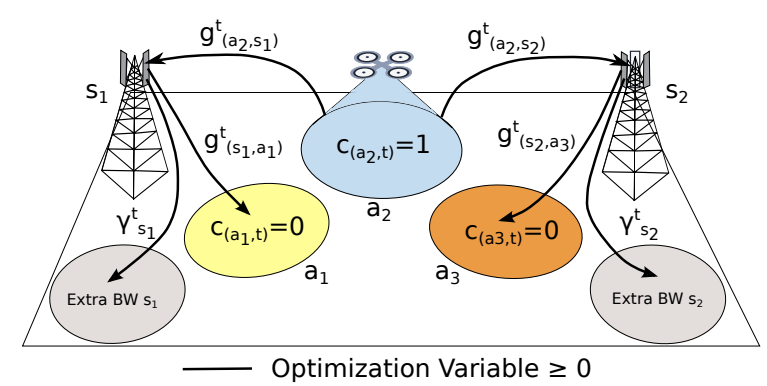

(a) BW variables $\geq 0$.

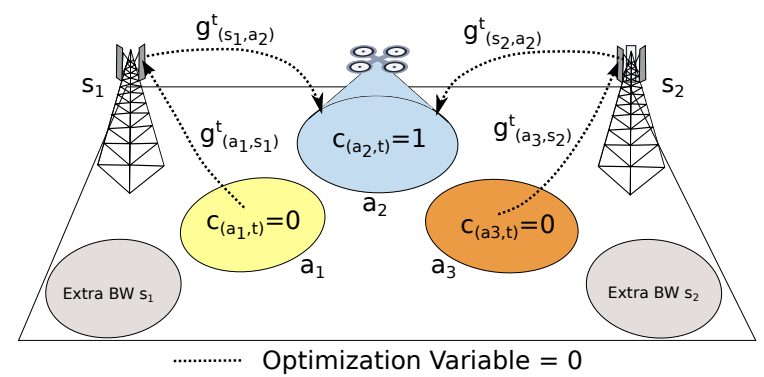

(b) BW variables forced to 0 .

Fig. 4. BW variables in a simple scenario in which a UAV-SC covers area $a_{2}$ at TS $t$.

$D_{\left(a_{3}, s_{1}\right)}=0, D_{\left(a_{3}, s_{2}\right)}=1$. Therefore, according to (10), $g_{\left(a_{1}, s_{1}\right)}^{t}=0, g_{\left(a_{1}, s_{2}\right)}^{t}=0, g_{\left(a_{2}, s_{1}\right)}^{t} \geq 0, g_{\left(a_{2}, s_{2}\right)}^{t} \geq 0$, $g_{\left(a_{3}, s_{1}\right)}^{t}=0, g_{\left(a_{3}, s_{2}\right)}^{t}=0$. On the other hand, according to (11), $g_{\left(s_{1}, a_{1}\right)}^{t} \geq 0, g_{\left(s_{2}, a_{1}\right)}^{t}=0, g_{\left(s_{1}, a_{2}\right)}^{t}=0, g_{\left(s_{2}, a_{2}\right)}^{t}=0$, $g_{\left(s_{2}, a_{3}\right)}^{t} \geq 0, g_{\left(s_{1}, a_{3}\right)}^{t}=0$. Finally, according to (12) the flow $g_{\left(a_{2}, s_{1}\right)}^{t}$ can be redistributed to $g_{\left(s_{1}, a_{1}\right)}^{t}$ and $\gamma_{s_{1}}^{t}$. In a similar way, the flow $g_{\left(a_{2}, s_{2}\right)}^{t}$ can be redistributed to $g_{\left(s_{2}, a_{3}\right)}^{t}$ and $\gamma_{s_{2}}^{t}$.

Downlink Throughput Constraints. Let us denote a protocol overhead parameter as $O \in(0,1]$. In addition, we denote by $F_{a}^{\mathrm{UAV}-\mathrm{SC}}$ the average spectral efficiency of area $a \in A$ when it is served by a UAV-SC. On the other hand, we denote by $F_{(a, s)}^{\mathrm{MC}}$ the average spectral efficiency of area $a \in A$ when it is served by MC in site $s \in S$. The total throughput of area $a$ at TS $t \in T$ is then:

$$
\begin{aligned}
r_{(a, t)} & =O \cdot\left\{F_{a}^{\mathrm{UAV}-\mathrm{SC}} \cdot W_{a}^{\mathrm{UAV}-\mathrm{SC}} \cdot c_{(a, t)}\right. \\
& \left.+\sum_{s \in S} F_{(a, s)}^{\mathrm{MC}} \cdot\left[W_{(a, s)}^{\mathrm{BASE}} \cdot\left(1-c_{(a, t)}\right)+W_{s}^{\mathrm{TOT}} \cdot g_{(s, a)}^{t}\right]\right\} \\
& \forall a \in A, t \in T
\end{aligned}
$$

In the previous equation, we model the following options: i) the area is served by a UAV-SC, or ii) the area is served by (multiple) MCs. The coverage variable $c_{(a, t)}$ is used to distinguish each option. In particular, if the area is covered by the UAV-SC, only the term i) is accounted. On the contrary, if the area is not served by any UAV-SC, it is served by the $\mathrm{MC}(\mathrm{s})$, which include the baseline amount of BW, plus the (possible) additional BW drained from the areas served by UAV-SCs.

\subsection{Objective Function and Complete Model}

We consider a multi-objective function to jointly pursue: i) the maximization of the revenue from traffic thanks to the exploitation of the UAV-SCs, ii) the maximization of the revenue for selling energy to the grid and iii) the minimization of the costs for buying energy from the grid. Specifically, the throughput and energy terms are properly weighed by including the following parameters: i) throughput weight $R^{\text {RATE }}$,ii) energy sold weight $R_{t}^{\mathrm{SELL}}$, which varies over $t \in T$, iii) energy bought weight $C_{t}^{\mathrm{BUY}}$, which also varies over $t$. The overall objective function of the JOINT MANAGEMENT of Multi-Area Throughrut AND EnERgy (J-MATE) model is then:

$\max \sum_{t \in T}\left[R^{\mathrm{RATE}} \cdot \sum_{a \in A} r_{(a, t)}+\sum_{s \in S} R_{t}^{\mathrm{SELL}} e_{(s, t)}^{\mathrm{SELL}}-\sum_{s \in S} C_{t}^{\mathrm{BUY}} e_{(s, t)}^{\mathrm{BUY}}\right]$

under constraints: (1)-(13), with variables: $m_{(l, u)} \in\{0,1\}$, $c_{(a, t)} \in\{0,1\}, w_{(l, u)} \geq 0, b_{(u, t)} \geq 0, e_{(s, t)}^{\mathrm{BUY}} \geq 0, e_{(s, t)}^{\mathrm{SELL}} \geq 0$, $g_{(a, s)}^{t} \geq 0, g_{(s, a)}^{t} \geq 0, \gamma_{s}^{t} \geq 0, r_{(a, t)} \geq 0$.

Proposition 1. The J-MATE problem is NP-Hard.

Proof. We consider a special case of the J-MATE, denoted as $\mathrm{M}-\mathrm{COV}$, whose goal is to maximize the coverage of the areas by means of UAV-SCs in all the TSs. M-COV is defined as: $\max \sum_{a \in A} \sum_{t \in T} c_{(a, t)}$, subject to: (1), (2), (3), (4) under variables: $m_{(l, u)} \in\{0,1\}, c_{(a, t)} \in\{0,1\}$. It is possible to note that $\mathrm{M}-\mathrm{COV}$ is a variant of the multi-commodity problem with unsplittable (integral) flows, a type of problem known to be NP-Hard [28]. Since M-COV is included in J-MATE, we can conclude that also J-MATE is NP-Hard.

\section{BBSR ALGORITHM}

We develop a new algorithm, called BALANCE ENERGY BOUGHT, ENERGY SOLD AND THROUGHPUT REVENUE (BBSR) to practically solve the J-MATE problem. Alg. 1 reports the main steps of the BBSR algorithm. To ease the notation, we remove the indexes when the parameter/variable has to be passed/retrieved entirely from/to a routine of the algorithm. As suggested by its name, the main goal of BBSR is to balance the different terms of the objective function in (14), by selectively scheduling the UAVSCs missions, the BW release, the BW assignment, and the energy that is exchanged with the grid. To pursue this goal, BBSR applies a divide-et-impera approach, which is detailed below.

After the initialization of the internal variables (lines 1-7), BBSR considers the sorting of each site-area pair $(s, a)$ with a given rule (line 9). The idea of this step is to prioritize the areas that need to be covered by UAV-SCs. We consider the following sorting criteria for the $(s, a)$ pairs: i) increasing BW $W_{(a, s)}^{\mathrm{BASE}}$, ii) increasing spectral efficiency $F_{(a, s)}^{\mathrm{MC}}$, or iii) increasing rate $O \cdot W_{(a, s)}^{\mathrm{BASE}} \cdot F_{(a, s)}^{\mathrm{MC}}$. The selection of the sorting criterium is left as an algorithm option. In the following (lines 10-12), BBSR iterates over each pair $(s, a)$. More in depth, the algorithm selects the current area $a$ to be covered under the following conditions: i) $a$ receives $\mathrm{BW}$ from $s$, ii) $a$ is not covered by any UAV-SC and iii) the number of available UAV-SCs is larger than two (line 12). In the following, BBSR schedules the UAV-SCs missions to cover $a$ over the whole set of TSs (lines 13-18). More in depth, the algorithm selects the site $s_{2}$ used as recharging unit for the UAV-SC mission from $s_{2}$ to $a$. In BBSR, $s_{2}$ is the site with the minimum energy consumed by a UAV-SC moving from 


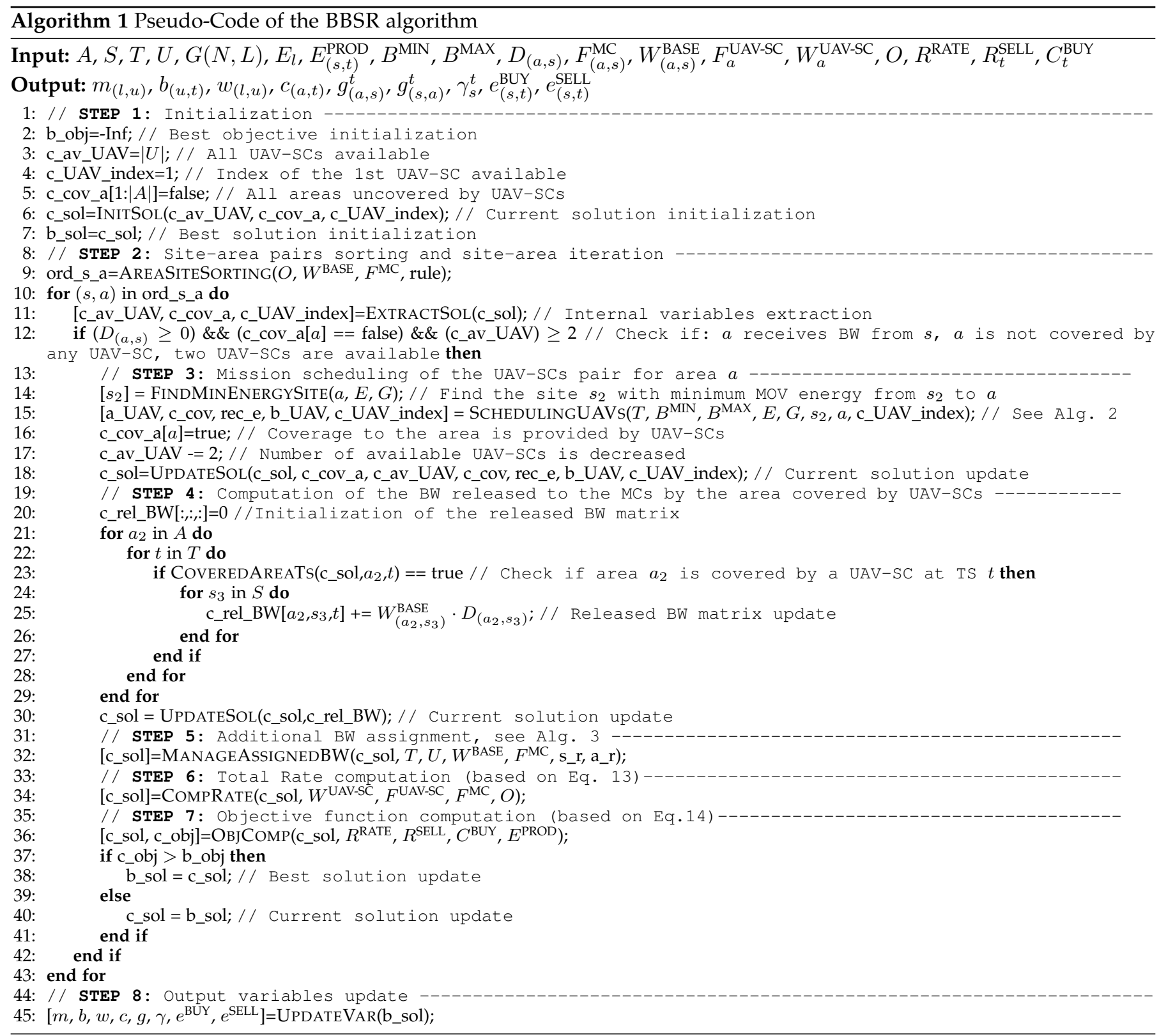

$s_{2}$ to $a$ (line 14). The algorithm then schedules the UAVSCs missions to cover $a$ from $s_{2}$ with the schedulingUAVs function (line 15), which is expanded in Alg.2. The implemented scheduling scheme exploits the intuition that one UAV-SC covers the area and another one is parked in $s_{2}$. Then, when the first UAV-SC needs to be recharged in $s_{2}$, the second one is moved from $s_{2}$ to $a$, in order to cover $a$ in the subsequent TSs. This mission scheme is then repeated across the whole set of TSs $t \in T$.

To give more insight, the SchedulingUAVs function of Alg.2 requires as input the current index C_UAV_index (which is used to keep track of the UAV-SCs that have been assigned so far to the areas), the set of TSs $T$, the minimum and maximum battery levels $B^{\mathrm{MIN}}$ and $B^{\mathrm{MAX}}$, the energy values in the multi-period graph $E_{l} \quad \forall l \in L$, the current site $s$ and the current area $a$. The routine then produces as output: i) matrix a_UAV, storing the actions of the two UAVSCs for each TS, ii) array C_cov, storing the coverage values for the current area (true if area $a$ is covered by a UAV-SC, false otherwise) for each TS, iii) array rec_e, storing the amount of energy demanded to the ground site $s$ by the two UAV-SCs in each TS, iv) matrix b_UAV, storing the battery levels of the two UAV-SCs in each TS, v) the updated value of C_UAV_index.

The main intuitions behind Alg.2 are the following ones. First, the default action for the two UAV-SCs is set to STAY (line 5), with full battery charge (line 6). Then, the first UAV-SC is moved over the area in the initial TS (line 8), and the UAV-SC battery level is updated (lines 9-11). In the following, the routine iterates over the set of TSs, by: i) covering the area with the first UAV-SC while ensuring the battery bounds (lines 17-22), ii) scheduling the moving and recharging actions for the first UAV-SC when a recharge is needed (lines 22-32), iii) scheduling the moving and coverage action for the second UAV-SC (lines 33-46), and iv) swapping the first and the second UAV-SC (lines 47-48). Clearly, the entire function aims at maximizing the coverage provided by the UAV-SCs pair. 


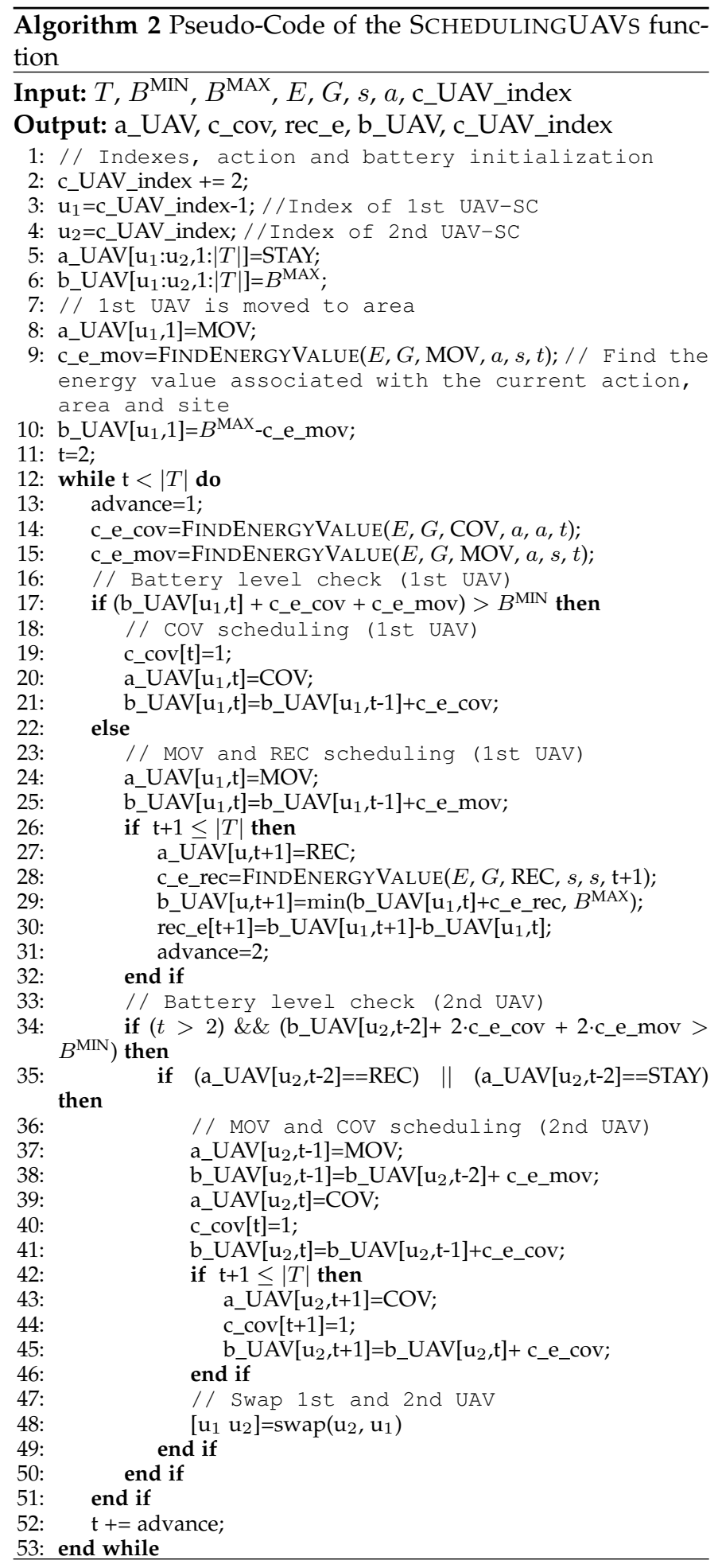

One specific aspect of Alg. 2 is the ability of scheduling the UAV-SC missions by always guaranteeing a safety margin in the battery level. For example, the battery level check on the first UAV-SC (line 17) always guarantees that the UAV-SC is able to cover the area in the current TS, and eventually come back to the site in the following TS. ${ }^{3}$ On

3. We remind that the values extracted by the FINDENERGYVALUE routine of Alg. 2 can be positive or negative, in accordance to the UAVSC action. When the action is REC the energy value is positive (i.e., the battery level is increased). When the action is MOV or COV the energy value is negative.

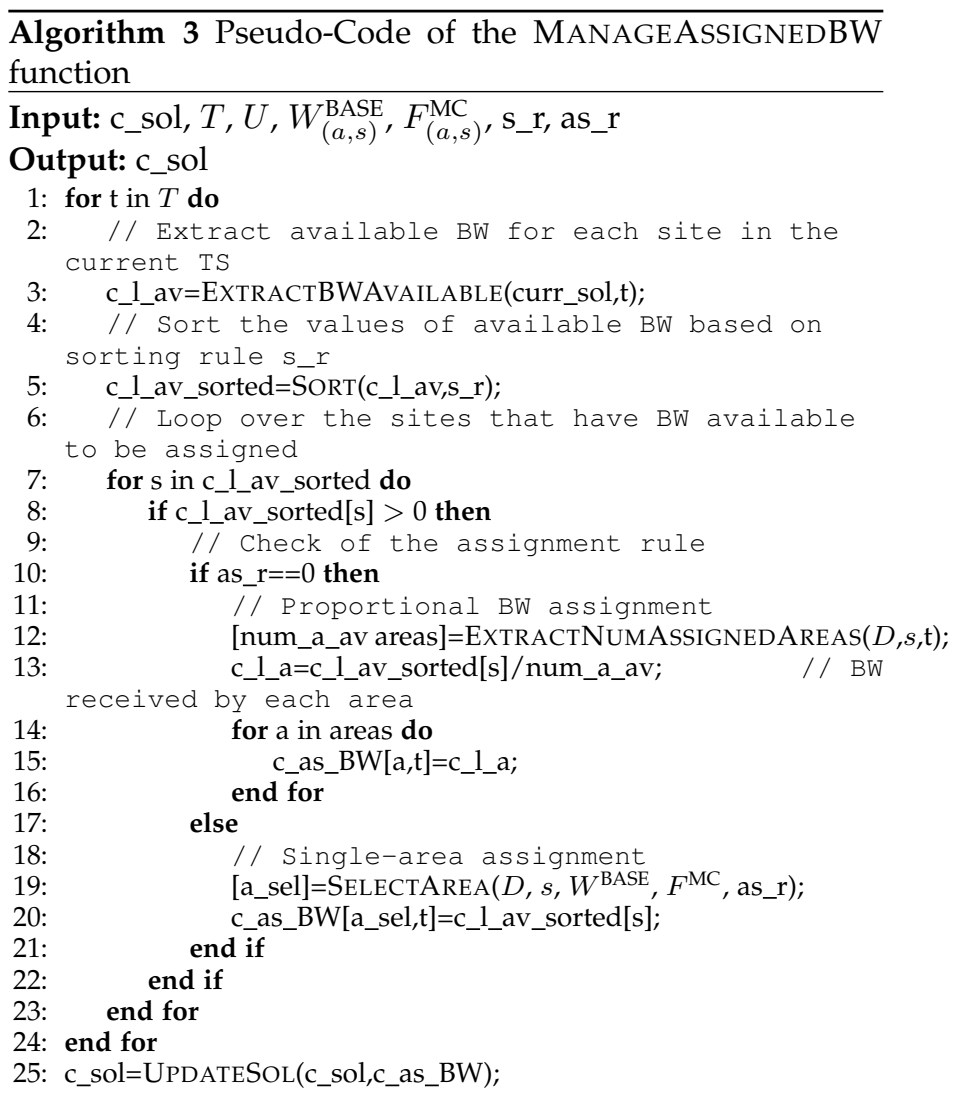

the other hand, the battery level check of the second UAVSC (line 34) is even more stringent, due to the fact that the second UAV-SC has to preliminary move from $s$ to $a$.

Returning to our description of the BBSR algorithm presented in Alg.1, the following parts of the mission scheduling step include: i) the update of the C_COV_a variable, which is set to true as the current area is served by UAV-SCs (line 16), the decrease of the number of available UAV-SCs (line 17), and the update of the solution with the variables modified so far (line 18). In the following step, the algorithm computes the amount of BW that is released in each TS to the sites by the areas covered by UAV-SCs (lines 19-29). The BW that has been released to the MCs is then assigned to the areas through the ManageAssignedBW function, which is expanded in Alg. 3. The function takes as input the current solution C_sol, the set of TSs $T$, the set of UAVSCs $U$, the baseline BW $W_{(a, s)}^{\mathrm{BASE}}$, the MC spectral efficiency $F_{(a, s)}^{\mathrm{MC}}$, a sorting rule s_r (ascending or descending), an assignment rule (proportional, BW decreasing, BW increasing, spectral efficiency increasing, spetral efficiency decreasing). The function then produces as output the updated solution (stored in the C_sol structure). The core of the function is the iteration over the set of TSs (lines 1-25) to: i) extract the available BW in each site, ii) sort the sites according to the available BW and the sorting rule (line 5), iii) iterate over the ordered set of sites (lines 7), iv) assign the available BW for each site (lines 8-22). Focusing on the last point, if the assignment rule is set to "proportional", the current available BW is equally split among all the areas currently served by the site (lines 10-16). Otherwise, a single area is selected, based on the sorting of baseline BW or spectral efficiency values (in increasing or decreasing order). Finally, 
the current solution is updated.

After the BW assignment, the last steps of Alg. 1 involve: i) the computation of the total rate provided to the areas, by applying Eq. 13 (line 34), ii) the computation of the objective function, based on Eq. 14 (line 36), iii) the update of best and current solutions (lines 37-42), and iv) the output of the solution variables (line 45 ).

\subsection{Computational Complexity}

We initially focus on the time complexity of the subroutines in Alg. 2 and Alg. 3. Specifically, the SCHEDULINGUAVs function in Alg. 2 has a complexity of $\mathcal{O}(|T|)$, due to the fact that it requires the scheduling of the actions for a pair of UAV-SCs in order to cover a given area over the whole set of TSs. In addition, we assume that the extraction of the current energy due to covering/moving in lines (14)-(15) of Alg. 2 is done in $\mathcal{O}(1){ }^{4}$

We then focus on the ManageAssignedBW function detailed in Alg. 3. More in depth, the extraction of the available BW from the current solution is done in line (3), resulting in a complexity of $\mathcal{O}(1)$. In the following, the BW values of the sites are sorted (line 5). This operation is done in $\mathcal{O}(|S| \log (|S|))$. Then, the following steps impose the assignement of the BW available on the sites to the areas (lines (6)-(23)), thus requiring $\mathcal{O}(|S| \cdot|A|)$ operations. Finally, the update of the current solution in line (24) can be done in $\mathcal{O}(1)$. Clearly, the operations in lines (2)-(23) are repeated over the whole set of TSs. Overall, the total complexity of the ManageAssignedBW function is $\mathcal{O}(|T| \cdot|S| \cdot(\log (|S|)+|A|))$.

Focusing then on the entire complexity of BBSR in Alg. 1, the initialization in lines (2)-(7) requires $\mathcal{O}(|A|)$ iterations. In addition, the area-site pairs sorting in line (9) is done in $\mathcal{O}(|S| \cdot|A| \log (|S| \cdot|A|))$. Then, for each sorted pair, the minimum energy site is selected (line 13), resulting in a complexity of $\mathcal{O}(|S|)$. In the following, the SchedulingUAVs function is invoked. Therefore, the complexity of this operation is $\mathcal{O}(|T|)$. The update of the solution in line (19) is done in $\mathcal{O}(1)$. In the following, the computation of the released BW is done in $\mathcal{O}(|S| \cdot|A| \cdot|T|)$. The update of the solution in line (30) requires again $\mathcal{O}(1)$. The ManageAssignedBW in line (32) has a complexity of $\mathcal{O}(|T| \cdot|S| \cdot(\log (|S|)+|A|))$. Finally, the rate computation (line 34) and objective function computation (line 36) require $\mathcal{O}(|T| \cdot|A|)$ and $\mathcal{O}(|T| \cdot(|A|+|S|))$, respectively. Overall, the total complexity of BBSR is in the order of $\mathcal{O}\left(|A| \cdot|S|^{2} \cdot|T| \cdot(|A|+\log (|S|))\right.$. Therefore, we can claim that the complexity of BBSR grows quadratically with the size $A$ and $S$, while only linearly with the size of $T$.

\section{Rotorua Scenario}

We divide the description of the scenario in the following subsections: i) areas, sites, coverage and signal levels, ii) spectral efficiency and bandwidth, iii) UAV-SC energy model, iv) site energy capabilities, and v) multi-period graph generation. We then provide more details about each of the aforementioned steps.

4. We recall that the current energy value for a UAV action at a given TS between two places can be directly retrieved from $E_{l}$ when the head $h(l)$ and the tail $t(l)$ of link $l$ are defined. In our case, we have $h(l)=$ $(a, t)$ and $t(l)=(a, t-1)$ when the action is COV, and $h(l)=(a, t)$ and $t(l)=(s, t-1)$ when the action is MOV.

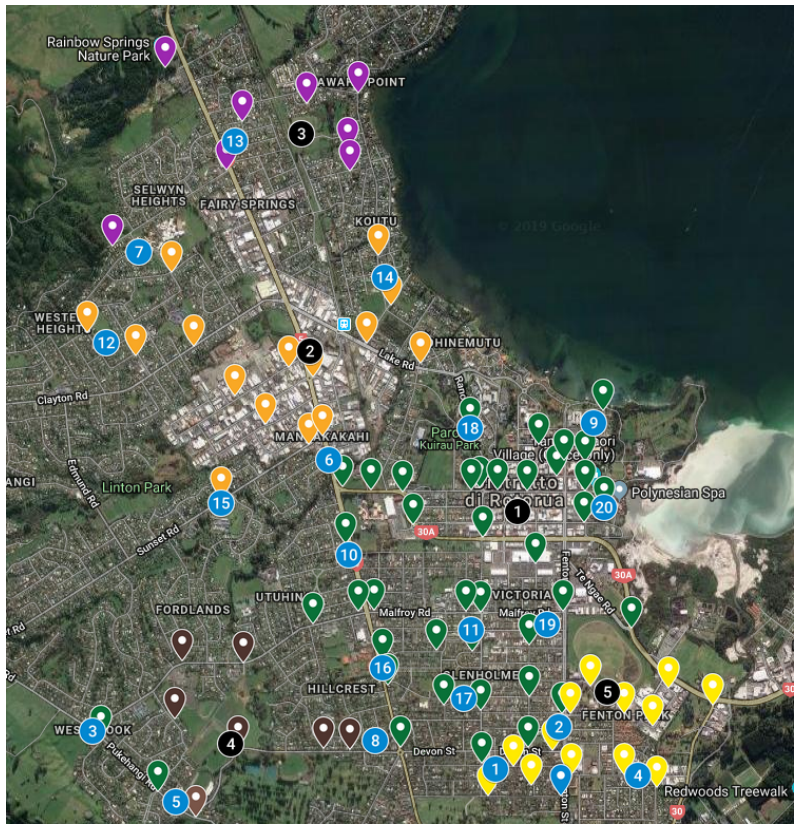

Fig. 5. Positions of MC sites (black circles), area centers (blue circles), CQI measurements (colored pins), and terrain view (source: Google Maps) in the Rotorua scenario, New Zealand. The colors of the CQI pins are set in accordance to the serving MC (Figure best viewed in colors).

\subsection{Areas, Sites, Coverage and Signal Levels}

We consider a rural scenario located in Rotorua, a town located in the North Island of New Zealand. Fig. 5 reports the terrain view of the scenario. In order to derive realistic sets to our problem, we measure different parameters from a real $4 \mathrm{G}$ network installed in Rotorua. In particular, we use the CellMapper application [29] installed on a Samsung S6 device and connected to the Vodafone NZ operator. CellMapper allows the generation of crowdsourced maps of MC locations, including the information about the coverage of each MC. To this aim, CellMapper performs continuous measurements, which are sent to the CellMapper cloud at regular intervals, in order to build detailed coverage maps that are made available to users through a web-based interface. In our case, we exploit the CellMapper option that allows saving the measurements locally on the smartphone. The saved measurements are then processed offline and used to generate our scenario. The data locally stored by the App include the current location (in GPS format), the Reference Signal Received Power (RSRP) metric, the operator's ID and the ID of the serving MC. More in depth, the RSRP metric is used by the operator as a proxy of the MCs coverage, e.g. for estimating the channel quality [30], typically in conjunction with other indexes. In our case, we record the RSRP measurements with CellMapper by walking with the smartphone over the main streets of Rotorua during two working days in January 2019. Fig. 6(a) reports the measured RSRP values. As expected, this metric is not constant over the territory. Specifically, the RSRP is in generally higher in proximity to the MC, and lower at the cell edges. By filtering the RSRP values from each single MC (see e.g., Fig.6(b)), we are able to: i) obtain the RSRP measurements for each BS, ii) find the $\mathrm{MC}$ location in proximity to the highest RSRP values (the 


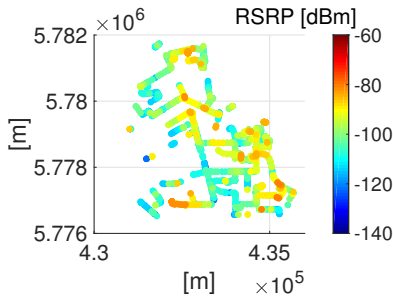

(a) All MCs

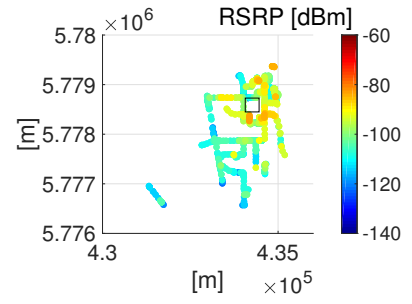

(b) Single MC Example
Fig. 6. RSRP coverage measurements over the Rotorua scenario, New Zealand. The square in (b) marks the position of the MC (Subfigures best viewed in colors).

exact positioning is then retrieved through a visual check performed in the candidate MC location).

We then select a set of $|S|=5$ sites, which are assumed to be co-located with the MCs of Vodafone NZ found through the analysis of RSRP measurements. Fig. 5 reports the positions of MC sites (in black circles). As expected, the $\mathrm{MC}$ sites are spread over the territory to globally cover the Rotorua town. In addition, we select a set of $|A|=20$ areas. The positions of the centers of the areas are shown in Fig. 5 with blue circles. The areas are selected according to the following principles: i) avoiding proximity between them, and ii) cover different neighborhoods of the town.

Given the RSRP values in each measurement point, we then build the coverage map of each MC as follows: we divide the territory with a regular grid, with squares of size equal to 500 [m]. This size is set to: i) avoid artifact coverage holes, which may appear due to the fact that our measurements are taken over the streets, and not inside courtyards, gardens and private streets, and ii) take into account that the coverage radius of a typical MC is in the order of different kilometers. For each square, we then count the numbers of RSRP measurements of the current MC in site $s \in S$ falling in the square. If the number of measurements is zero, then the current square is not covered by $s$. Otherwise, we assume that the current square is covered by $s$. We then denote with $\zeta_{s}\left[\mathrm{~km}^{2}\right]$ the total area covered by $s \in S$, which is computed as the summation of the areas of the squares covered by $s$. Finally, we assume that the areas $a \in A$ are circles with a radius of $250[\mathrm{~m}]$ - a value in line with the coverage of currently deployed SCs [31]. We then denote the total area size as $\zeta_{a}$, which is equal to $0.19\left[\mathrm{~km}^{2}\right] \quad \forall a \in A$.

In the following step, we consider the measurements of the level of the Quality of Service (QoS) provided to users. To this aim, we perform a set of measurements of the Channel Quality Indicator (CQI) values at fixed locations of the Rotorua scenario. The CQI is read on the CellMapper interface after standing in the same location for a sufficiently long period of time (e.g., 2-3 minutes), to avoid side effects like fading and/or changes in the sight conditions w.r.t. the serving MC. The locations of the measurements points in the scenario are reported in Fig. 5 with colored pins, where each pin is colored in accordance to the serving MC. From the figure, we can observe that: i) the measurements are sufficiently spread over the territory, with an higher density in the town center (mid-right part of the figure), ii) CQI measurements from all the MCs in the scenario are collected. The CQI values obtained in this step are then used to compute the average spectral efficiency values associated with each area, as reported in the following subsection.

\subsection{Spectral Efficiency and Bandwidth}

We initially detail the steps to retrieve the spectral efficiency values from the CQI measurements. We recall that our problem requires as input: i) the spectral efficiency $F_{(a, s)}^{\mathrm{MC}}$ when area $a$ is served by MC in site $s$, ii) the spectral efficiency $F_{a}^{\mathrm{UAV}-\mathrm{SC}}$ when area $a$ is served by a UAV-SC. In order to obtain the $F_{(a, s)}^{\mathrm{MC}}$ values, we proceed as follows: i) we compute the average of the CQI measurements from $s$ falling in the coverage radius of $a$, ii) we apply the CQI - spectral efficiency conversion table of [32] to the values of i). ${ }^{5}$ The obtained values of $F_{(a, s)}^{\mathrm{MC}}$ are reported in Tab. 3. Interestingly, we can note that: i) the spectral efficiency notably varies across the areas, ii) the same area is subject to different $F_{(a, s)}^{\mathrm{MC}}$ values when it is served by multiple MCs (see e.g., area 1 or area 2). Focusing on the spectral efficiency when the area is served by a UAV-SC, we set $F_{a}^{\mathrm{UAV}-\mathrm{SC}}=3.9[\mathrm{bps} / \mathrm{Hz}]$. This value corresponds to the maximum efficiency, which is assumed to be achieved thanks to the good channel conditions experienced by the users in the area in this case (i.e., Line of Sight and proximity to the SC).

In the next part, we compute the values of baseline BW assigned to each area. By assuming a fair BW assignment among the MCs serving the same area $a$, the baseline BW provided to area $a$ by MC in site $s$ is computed as:

$$
W_{(a, s)}^{\mathrm{BASE}}=W_{s}^{\mathrm{TOT}} \cdot \frac{\zeta_{a}}{\eta_{a} \cdot \zeta_{s}}
$$

where $W_{s}^{\mathrm{TOT}}=20$ [Mhz] (i.e., a typical setting for an MC), $\zeta_{a}$ is the area size, $\eta_{a}$ is the number of MCs serving $a$, and $\zeta_{s}$ is the total portion of territory covered by MC in site $s$. The obtained values of $W_{(a, s)}^{\mathrm{BASE}}$ are reported in Tab. 4. By observing the values in the table, we can observe that there is a strong heterogeneity of $W_{(a, s)}^{\mathrm{BASE}}$ values across the different areas, and even across the different MCs serving the same area. In any case, the values of baseline BW are not very large (i.e., much lower compared to the entire MC BW $\left.W_{s}^{\mathrm{TOT}}\right)$, thus suggesting that there is ample room to improve the throughput thanks to the exploitation of the UAV-SCs. To this aim, we assume a typical SC BW configuration when a UAV-SC serves an area, resulting in $W_{a}^{\mathrm{UAV}-\mathrm{SC}}=5[\mathrm{MHz}]$.

\subsection{UAV-SC energy model}

We consider here the parameters related to UAV-SC energy consumption. We start from the energy model of [26] to include the different contributions, namely: i) the amount of energy that is spent by the UAV-SC on the horizontal component (i.e., the level flight energy consumption), ii) the amount of energy that is spent by the UAV-SC on the vertical component (i.e., climbing/descending energy consumption). In addition, we also introduce to the model of [26] the contribution of energy when the UAV-SC activates the SC functionalities (i.e., during the area coverage).

More formally, we recall that the energy consumption $E_{l}$ is a weight associated with link $l \in L$ of the multiperiod graph, which is computed as the summation of

5. In case of non integer average CQI values, we apply a weighted average between upper and lower integer numbers. 
TABLE 3

Area spectral efficiency $F_{(a, s)}^{\mathrm{MC}}[\mathrm{bps} / \mathrm{Hz}]$ - Rotorua Scenario.

\begin{tabular}{|c|c|c|c|c|c|c|c|c|c|c|c|c|c|c|c|c|c|c|c|c|c|}
\hline & \multicolumn{20}{|c|}{ Area ID } \\
\hline & & 1 & 2 & 3 & 4 & 5 & 6 & 7 & 8 & 9 & 10 & 11 & 12 & 13 & 14 & 15 & 16 & 17 & 18 & 19 & 20 \\
\hline \multirow{5}{*}{ 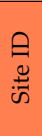 } & 1 & 0.6 & 2.3 & 1.7 & - & 1.7 & - & - & 0.4 & 2.7 & 0.8 & 1.9 & - & - & - & - & 2.1 & 1.2 & 0.2 & 1.3 & 1.8 \\
\hline & 2 & - & - & - & - & - & 1.9 & - & - & - & - & - & 2.3 & - & 1.7 & 1.3 & - & - & - & - & - \\
\hline & 3 & - & - & - & - & - & - & 2.3 & - & - & - & - & - & 0.2 & - & - & - & - & - & - & - \\
\hline & 4 & - & - & - & - & 1.9 & - & - & 2.7 & - & - & - & - & - & - & - & - & - & - & - & - \\
\hline & 5 & 1.5 & 1.9 & - & 1.7 & - & - & - & - & - & - & - & - & - & - & - & - & - & - & - & - \\
\hline
\end{tabular}

TABLE 4

Baseline BW $W_{(a, s)}^{\mathrm{BASE}}[\mathrm{MHz}]$ for each site and each area in the Rotorua Scenario.

\begin{tabular}{|cc|c|c|c|c|c|c|c|c|c|c|c|c|c|c|c|c|c|c|c|c|}
\hline & & 1 & 2 & 3 & 4 & 5 & 6 & 7 & 8 & 9 & 10 & 11 & 12 & 13 & 14 & 15 & 16 & 17 & 18 & 19 & 20 \\
\hline & 1 & 0.3 & 0.3 & 0.6 & - & 0.3 & - & - & 0.3 & 0.6 & 0.6 & 0.6 & - & - & - & - & 0.6 & 0.6 & 0.6 & 0.6 & 0.6 \\
\multirow{2}{*}{} & 2 & - & - & - & - & - & 0.7 & - & - & - & - & - & 0.7 & - & 0.7 & 0.7 & - & - & - & - & - \\
¡े & 3 & - & - & - & - & - & - & 1.4 & - & - & - & - & - & 1.4 & - & - & - & - & - & - & - \\
& 4 & - & - & - & - & 0.9 & - & - & 0.9 & - & - & - & - & - & - & - & - & - & - & - & - \\
& 5 & 0.9 & 0.9 & - & 1.8 & - & - & - & - & - & - & - & - & - & - & - & - & - & - & - & - \\
\hline
\end{tabular}

the different energy components, depending on the action associated with $l$. Let us denote with $E_{l}^{\mathrm{F}}, E_{l}^{\mathrm{V}}, E_{l}^{\mathrm{SC}}$ the level flight energy consumption, the climbing/descending energy consumption, and the SC energy consumption, respectively.

When the UAV-SC performs a MOV action on link $l$, the total energy consumption $E_{l}$ is:

$$
E_{l}=E_{l}^{\mathrm{F}}+E_{l}^{\mathrm{V}}, \quad \forall l \in L^{\mathrm{MOV}}
$$

On the other hand, when the UAV-SC performs a COV action on link $l, E_{l}$ becomes equal to:

$$
E_{l}=E_{l}^{\mathrm{F}}+E_{l}^{\mathrm{SC}}, \quad \forall l \in L^{\mathrm{COV}}
$$

Moreover, we assume that no energy is consumed when the UAV-SC is parked in a ground site or when the UAVSC activates the links from the fictitious nodes $\Omega$ and $\Phi$ More formally, we have: $E_{l}=0 \quad \forall l \in\left\{L^{\text {STAY }} \cup L^{\Omega} \cup L^{\Phi}\right\}$. Focusing on REC actions, we set $E_{l}=1000$ [Wh] $\forall l \in$ $L^{\mathrm{REC}}, B^{\mathrm{MAX}}=1000[\mathrm{Wh}], B^{\mathrm{MIN}}=100[\mathrm{Wh}]$, as in [12].

In the following, we provide more insights about the formal definitions of $E_{l}^{\mathrm{F}}, E_{l}^{\mathrm{V}}$ and $E^{\mathrm{SC}}$. The level flight energy consumption $E_{l}^{\mathrm{F}}$ is defined as:

$$
\begin{array}{r}
E_{l}^{\mathrm{F}}=\frac{(m \cdot g)^{2}}{\sqrt{2} \delta \sigma} \frac{1}{\sqrt{H_{l}^{(2)}+\sqrt{H_{l}^{(4)}+\left(\frac{m \cdot g}{\sigma}\right)^{2}}}} \Delta_{t}, \\
l \in L^{M O V} \cup L^{C O V}
\end{array}
$$

where $m, g, \delta, \sigma$ and $\Delta_{t}$ are the UAV-SC mass, the gravitational acceleration, the air density, the area of the UAV-SC rotor disks and the TS duration, respectively. In addition, $H_{l}$ denotes the horizontal speed, which is computed as:

$$
H_{l}=\frac{\epsilon_{\left(p_{1}, p_{2}\right)}}{\Delta_{t}}, \forall l: h(l)=\left(p_{2}, t\right), t(l)=\left(p_{1}, t\right)
$$

where $\epsilon_{\left(p_{1}, p_{2}\right)}$ is the distance between place $p_{1}$ and place $p_{2}$. In our scenario, we adopt the following settings: $m=12[\mathrm{~kg}], g=9.81\left[\mathrm{~m} / \mathrm{s}^{2}\right], \delta=1.225\left[\mathrm{~kg} / \mathrm{m}^{3}\right][26]$, $\sigma=3.141\left[\mathrm{~m}^{2}\right]^{6}{ }^{6} \epsilon_{\left(p_{1}, p_{2}\right)}$ based on the real positions of the places shown in Fig. 5, and $\Delta_{t}=600$ [s] $\forall t \in T$. Moreover, when the UAV-SC performs a COV action, it hovers over the center of area $a$. In this condition, it holds that $H_{l}=0, \quad \forall l \in L^{C O V}$.

6. With this setting, a rotor disk of diameter equal to $2[\mathrm{~m}]$ is assumed.
We then consider the climbing/descending energy consumption $E_{l}^{\mathrm{V}}$, which is defined as:

$$
E_{l}^{\mathrm{V}}=m \cdot g \cdot V_{l} \cdot \Delta_{t}, \quad l \in L^{M O V}
$$

where $V_{l}$ is the vertical speed on link $l$. If the UAV-SC is climbing, then $V_{l} \geq 0$. Otherwise, if the UAV-SC is descending, $V_{l} \leq 0$. In our scenario, the UAV-SC climbs from the ground level to the cruise altitude when performing a MOV action from a site to an area. On the contrary, the UAV-SC descends from the cruise altitude to the ground level when performing a MOV action from an area to a site. $V_{l}$ is then formally expressed as:

$$
V_{l}= \begin{cases}\frac{\kappa}{\Delta_{t}} & \text { if } \quad l \in L^{M O V}, p_{1} \in S, p_{2} \in A, \\ -\frac{\kappa}{\Delta_{t}} & \text { if } \quad l \in L^{M O V}, p_{1} \in A, p_{2} \in S,\end{cases}
$$

where $\left(p_{1}, t_{1}\right)=t(l),\left(p_{2}, t_{2}\right)=h(l), t_{1}=(t-1), t_{2}=t$, $\kappa=200[\mathrm{~m}]$ is the cruise altitude, which is again set in accordance to a typical UAV-SC setting [5].

Finally, we take into account the SC energy consumption $E_{l}^{\mathrm{SC}}$, which is denoted as:

$$
E_{l}^{\mathrm{SC}}=P_{t}^{\mathrm{SC}} \cdot \Delta_{t}, \quad \forall l \in L^{C O V}
$$

where $P_{t}^{\mathrm{SC}}=200[\mathrm{~W}]$ is the power consumed by SC functionalities, chosen in accordance to a realistic setting (see e.g., [33] with one transmission node).

\subsubsection{Model Validation}

Up to this point, a natural question is then: How accurate is the UAV-SC energy model used in this work? To verify this issue, we consider a simple (yet representative) testbed, by exploiting a DJI Phantom 3 Pro UAV with the following features: $m=1.28[\mathrm{~kg}], B^{\mathrm{MAX}}=68[\mathrm{Wh}], \sigma=0.0452\left[\mathrm{~m}^{2}\right]$. In addition, we consider the activation of the camera as an additional source of power to mimic the BS behavior, resulting in $P_{t}^{\mathrm{SC}}=50[\mathrm{~W}]$. We then perform the following tests: T1) climb up to $\kappa=12[\mathrm{~m}]$, then fly for $\Delta_{t}=300[\mathrm{~s}]$ for a total distance of $\epsilon_{\left(p_{1}, p_{2}\right)}=1440$ [m] ("Horizontal Flight"); T2) activate the camera recording, climb up to $\kappa=12[\mathrm{~m}]$, then fly for $\Delta_{t}=300[s]$ for a total distance of $\epsilon_{\left(p_{1}, p_{2}\right)}=1920[\mathrm{~m}]$ ("Horizontal Flight + Recording"); T3) climb up to $\kappa=3$ [m], then hover for $\Delta_{t}=300[s]$ ("Hovering"); T4) climb up to $\kappa=104$ [m] with $\Delta_{t}=70$ [s], then descend to ground with 


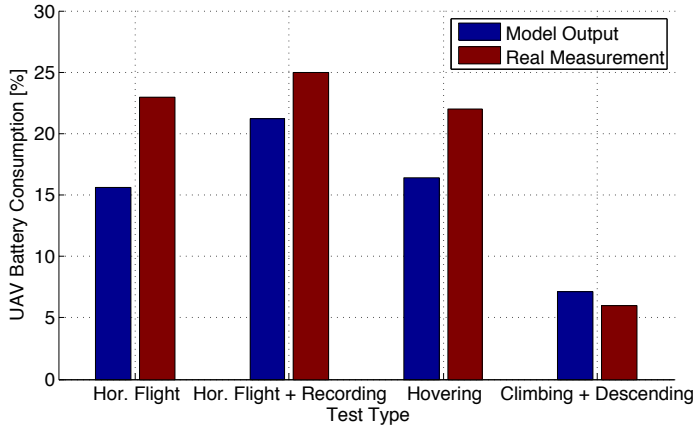

Fig. 7. Comparison between the real measurements and the output of the UAV-SC energy model under different test conditions.

$\Delta_{t}=60$ [s]. For each test, we measure the initial and final battery level percentage. In addition, we plug the input data to our UAV-SC energy model. Fig. 7 reports the comparison between the real measurements and the output of the model for each test. Although some variations are clearly visible, the outcome of the model is generally in line with the one obtained from the real measurements. For example, the climbing and descending test consumes less battery compared to the other ones. This is due to the fact that: i) this test requires less time to be completed, and ii) the UAV consumes less energy when it is descending. In addition, the largest battery consumption is recorded when the UAV activates the camera, which brings an additional energy factor (properly taken into account by our model).

\subsection{Site Energy Capabilities}

We then consider the setting of parameters related to the energy managed by the sites. We assume an SP plant of size equal to $10[\mathrm{kWp}]$ deployed in each site. In addition we set $|T|=144$ TSs over $24 \mathrm{~h}$, resulting in $\Delta_{t}=600[\mathrm{~s}]$. $^{7}$ Moreover, we select one day in December (corresponding to the summer in the Southern hemisphere). We then plug the day, the position location, the size of the SP plant and the day in the PVWatts calculator [34] to obtain the SP energy production over time. Without loss of generality, we assume that all the sites adopt the same SP energy production profile. The obtained values of $E_{(s, t)}^{\mathrm{PROD}}$ for each TS are then shown in Fig. 8. As expected, the energy production is positive during the day and zero during the night.

In the following, we consider the setting of the revenue from the energy sold $R_{t}^{\mathrm{SELL}}$, which is set equal to 0.07 [NZD/KWh] $\forall t \in T$ in accordance to [35]. We then set the cost for the energy bought $C_{t}^{\mathrm{BUY}}$ from the NZ electricity authority [36]. By observing the trends of $R_{t}^{\mathrm{SELL}}$ and $C_{t}^{\mathrm{BUY}}$, reported in Fig. 8, we can note that $C_{t}^{\mathrm{BUY}}>R_{t}^{\mathrm{SELL}} \quad \forall t \in T$ (as expected).

\subsection{Multi-Period Graph Generation}

We then set the parameters related to the multi-period graph, which is used to model the UAV-SC missions over space and time. More in depth, we consider a set of $|D|=2 \cdot|A|=40$ UAV-SCs. In this way, we guarantee that each area can be potentially covered by a UAV-SC in each

7. This setting is in accordance with the $\Delta_{t}$ values used in the UAVSC energy model of Sec. 6.3.

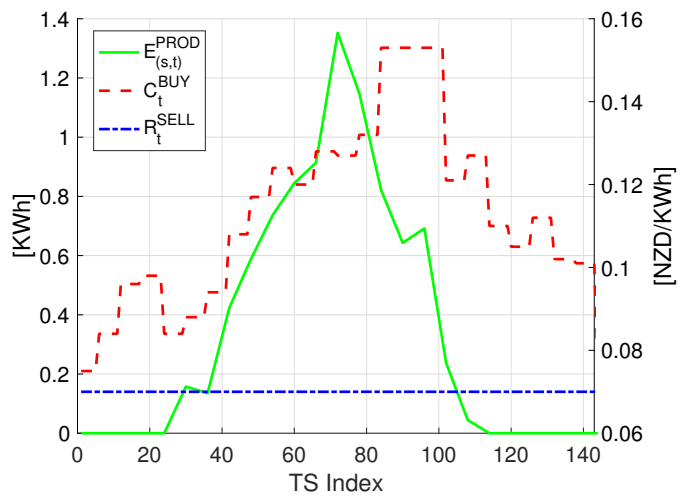

Fig. 8. Energy produced vs. time $E_{(s, t)}^{\mathrm{PROD}}[\mathrm{KWh}]$ for each site $s \in S$, revenue from the energy sold vs. time $R_{t}^{\mathrm{SELL}}[\mathrm{NZD} / \mathrm{KWh}$, and cost for the energy bought vs. time $C_{t}^{\mathrm{BUY}}$ [NZD/KWh].

TS. In addition, we avoid an excessive number of UAV-SCs, which would otherwise increase too much the number of flow variables $m_{(l, u)}$.

We then build the multi-period graph $G(N, L)$ by considering as nodes $N$ the pairs $(p, t)$ where $p \in S \cup A$ and $t \in T$. In addition, we add to $N$ also the fictitious nodes $\Omega$ and $\Phi$. Focusing on the links $l \in L$, the energy of each link $E_{l}$ is set in accordance to the link type ( $L^{\mathrm{MOV}}, L^{\mathrm{COV}}, L^{\mathrm{REC}}$, $\left.L^{\text {STAY }}, L^{\Omega}, L^{\Phi}\right)$, as detailed in Sec. 6.3. Moreover, in order to prevent an excessive number of links in $G$, we prune the original graph by removing all the links between two places $p_{1}$ and $p_{2}$ meeting one of the following criteria: i) distance $\epsilon_{\left(p_{1}, p_{2}\right)}>1600[\mathrm{~m}]$ and ii) $p_{1}=s_{1} \in S, p_{1}=s_{2} \in S$, $s_{1} \neq s_{2}$, iii) $p_{1}=a_{1} \in A, p_{1}=a_{2} \in A, a_{1} \neq a_{2}$. In this way, i) we avoid an excessive distance travelled by a UAV-SC from a site to an area, ii) a UAV-SC serving an area has to come back to a site before serving an area (while the serving of the same area across a set of consecutive TSs is allowed), and iii) when moving from a site to (another) one, at least one area has to be visited during the mission. The resulting graph is then composed of $|N|=3602$ nodes and $|L|=16456$ links.

\section{Results}

We code the J-MATE model as a $\mathrm{C}++$ program, which is run by CPLEX v.12.8 on a server machine equipped with a 3.5 [GHz] Xeon 8-Cores CPU and 64 [GB] of RAM. The BBSR algorithm is coded in Matlab and run on the same server machine with the software Matlab 2017. We also consider the following stopping criteria for the optimization of J-MATE: i) a maximum time limit of 24 [hours], and i) a MIP gap tolerance $\leq 2 \%$. In this way, J-MATE returns the current solution if either condition i) or ii) is verified. Focusing on BBSR, we set the following algorithm parameters: sitearea pairs sorted by increasing $W_{(a, s)}^{\mathrm{BASE}}$ values (Alg.1, line 9), site sorting based on decreasing available BW (Alg.3, line 5), assigned BW based on a proportional rule (Alg.3, line 10). ${ }^{8}$ We then run the J-MATE model and the BBSR algorithm over the Rotorua scenario. In order to add two

8. The selected combination of parameters achieved the best performance compared to the other possible ones (not reported here due to the lack of space.) 


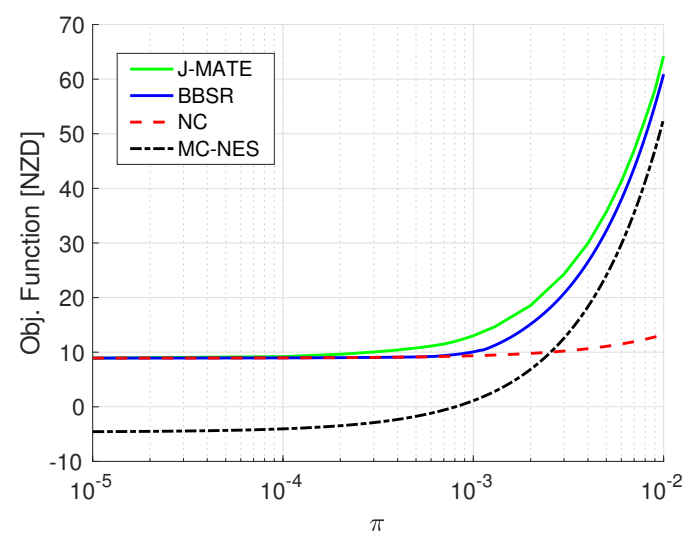

Fig. 9. Objective function variation for J-MATE, BBSR, NC and MCNES vs. the throughput revenue $R^{\mathrm{RATE}}(|T|=24)$.

terms of comparison, we consider the following strategies: i) MAXIMUM UAV-SC COVERAGE - NO ENERGY SOlD (MCNES), which targets the maximization of the areas covered by the UAV-SCs over the set of TSs, without selling any energy to the grid, and ii) NO UAV-SC COVERAGE (NC), which instead always assumes that the areas are served solely by the MCs. In this way, all the UAV-SCs are always in the STAY state and the whole energy produced by SPs is sold to the grid using this strategy.

\subsection{Objective Function Variation}

Since running J-MATE with $|T|=144$ TSs is computationally infeasible (due to the size of the problem and its NPHardness nature), we consider a subset of $|T|=24$ TSs, from $t_{1}=61$ to $t_{2}=84$, i.e., the central hours of SP energy production in Fig. 8. We then run J-MATE, BBSR, MC-NES and NC over the set of 24 TSs. Fig. 9 reports the values of the objective function of Eq. (14), which is produced as output by J-MATE, BBSR, and computed with a postprocessing step with MC-NES and NC. The figure shows the impact of the throughput weight $R^{\text {RATE }}$ on the total objective. Specifically, when $R^{\text {RATE }}$ is low (left part of the figure), there is no revenue from serving the areas with the UAV-SCs. Consequently, no UAV-SC is used by J-MATE and BBSR. In this case, all the energy is sold to the grid, thus realizing a revenue for the operator. The obtained gain is the maximum one since the two strategies overlap with NC. On the other hand, the MC-NES strategy introduces a cost loss in this case, since maximizing the UAV-SC coverage has a negative impact on the objective function. When the throughput $R^{\text {RATE }}$ is increased (from left to right part of the figure), we can see that all the strategies tend to increase the objective function (as expected). However, the best strategy turns out to be J-MATE, which takes explicitly into account all the revenues/costs terms. In addition, BBSR performs pretty close to J-MATE. Clearly, for large values of $R^{\text {RATE }}$ (right part of the figure), the NC strategy turns out to be the worse one. This is an expected result, as in this case serving an area with a UAV-SC brings a substantial increase in the throughput, and hence an increase in the objective function. Since NC does not exploit any UAV-SC, the gap compared to the other strategies is very large. Overall, the results confirm that J-MATE and BBSR are always able to
TABLE 5

Computational comparison of J-MATE and BBSR $(|T|=24)$.

\begin{tabular}{|c|c|c|c|c|c|}
\hline & \multirow{2}{*}{ Metric } & \multicolumn{4}{|c|}{$R^{\text {RATE }}[\mathrm{NZD} / \mathrm{Mbps}]$} \\
\hline & & $10^{-5}$ & $10^{-4}$ & $10^{-3}$ & $10^{-2}$ \\
\hline \multirow{3}{*}{ 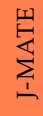 } & Time & $6.19[\mathrm{~s}]$ & $774.71[\mathrm{~s}]$ & $62278.57[\mathrm{~s}]$ & $60192.36[\mathrm{~s}]$ \\
\hline & Memory & N.A. & $288.26[\mathrm{MB}]$ & 67765.89 [MB] & $8431.71[\mathrm{MB}]$ \\
\hline & MIP gap & $0.46 \%$ & $1.91 \%$ & $5.15 \%$ & $1.91 \%$ \\
\hline $\begin{array}{l}\text { 㟧 } \\
\text { m }\end{array}$ & Time & \multirow{2}{*}{\multicolumn{4}{|c|}{$\begin{array}{c}<1[\mathrm{~s}] \\
.44[\mathrm{MB}]\end{array}$}} \\
\hline & Men & & & & \\
\hline
\end{tabular}

achieve the best solutions in terms of objective function, for any given value of $R^{\text {RATE }}$. This is an important outcome, as the actual value of $R^{\mathrm{RATE}}$ is chosen by the operator, based on its policies, e.g., maximizing the throughput revenues, minimizing the energy costs, or a mixture between the previous two terms.

\subsection{Computational Performance}

In the following, we describe the computational metrics considered to evaluate the performance of J-MATE and BBSR. Focusing on J-MATE, we consider: i) the time to retrieve the best solution, ii) the virtual memory occupation (reported by CPLEX), and iii) the MIP gap of the best solution found. Focusing on BBSR, we take into account: i) the time to run the algorithm, and ii) the virtual memory occupation (reported by Matlab). Tab. 5 reports the obtained results vs. a set of representative values of $R^{\text {RATE}}$. Several considerations hold in this case. First, when $R^{\text {RATE }}$ is low (left part of the table), solving the optimization problem is in general easy, as the best solution does not exploit UAV-SCs. Second, when $R^{\mathrm{RATE}}$ is increased, the problem starts exploiting the UAV-SCs to cover areas. Consequently, we can note a huge increase of the considered metrics (time, memory and mipgap). Third, when $R^{\text {RATE }}$ assumes the intermediate value of $10^{-3}$ [NZD/Mbps], the problem is even more challenging to be solved, since all the virtual memory available by the server is occupied, and the program has to be manually stopped. Fourth, when $R^{\mathrm{RATE}}$ is further increased (right part of the table), J-MATE still requires large resources to be solved. Focusing instead on BBSR, both the time to retrieve the solution and the memory occupation are always very low, thus proving the computational efficiency of our heuristic. Moreover, the metrics are not affected by the variation of $R^{\text {RATE }}$. This is an expected result, as $R^{\text {RATE }}$ does not influence the algorithm complexity (see Sec. 5.1).

\subsection{Rate, Energy, and Fairness Comparison}

We then consider the full set of TSs $|T|=144$ and we run the BBSR, MC-NES and NC algorithms. We perform an investigation of the impact of the weight $R^{\text {RATE }}$ under a set of performance metrics. More in depth, we initially consider: i) the total data rate (denoted as $\rho^{\mathrm{TOT}}$ ), ii) the balance between the energy revenues and the energy costs (denoted as $B^{\mathrm{ENERGY}}$ ), iii) the Jain's fairness index on the average rate per area over time (denoted as $J^{\mathrm{RATE}}$ ). The equations to compute $\rho^{\mathrm{TOT}}, B^{\mathrm{ENERGY}}$ and $J^{\mathrm{RATE}}$ are detailed in Tab. 6. Fig. 10(a) reports $\rho^{\mathrm{TOT}}$ for the different strategies. As expected, the lower bound on $\rho^{\mathrm{TOT}}$ is achieved by $\mathrm{NC}$, since this strategy does not use any UAV-SC. On the other 
TABLE 6

Performance Metrics.

\begin{tabular}{|c|c|}
\hline Metric & Expression \\
\hline Total Data Rate & $\rho^{\mathrm{TOT}}=\sum_{t \in T} \sum_{a \in A} r_{(a, t)}$ \\
\hline Energy Balance & $B^{\mathrm{ENERGY}}=\sum_{t \in T} \sum_{s \in S}\left(R_{t}^{\mathrm{SELL}} e_{(s, t)}^{\mathrm{SELL}}-C_{t}^{\mathrm{BUY}} e_{(s, t)}^{\mathrm{BUY}}\right)$ \\
\hline Rate Fairness & $J^{\mathrm{RATE}}=\frac{\left(\sum_{a \in A} \hat{r}_{a}\right)^{2}}{\left(|A| \cdot \sum_{a} \in \hat{r}_{a}^{2}\right)}$ \\
$\hat{r}_{a}=\frac{\sum_{t \in T} r_{(a, t)}}{|T|}$
\end{tabular}

hand, the upper bound is achieved by MC-NES, thanks to the coverage provided by the UAV-SCs over the areas. In addition, the ratio between upper and lower bound is more than one order of magnitude, since each UAV-SC covering an area introduces a joint increase of spectral efficiency and BW. Interestingly, BBSR presents an increasing trend between lower and upper bounds, depending on the values of $R^{\text {RATE }}$.

Fig. 10(b) reports then the values of $B^{\text {ENERGY }}$. In this case, we can observe an inverse trend compared to $\rho^{\text {TOT }}$. Specifically, the best strategy is now NC, which sells all the energy produced by SPs to the grid. The MC-NES strategy requires instead an amount of energy bought from the grid, and therefore $B^{\text {ENERGY }}<0$. Again, BBSR is able to adapt between the two aforementioned conditions. Finally, the $J^{\text {RATE }}$ metric is shown in Fig. 10(c). Three consideration hold: i) $J^{\text {RATE }}$ is maximum with MC-NES, due to the fact that all the areas receive the same throughput, thanks to the UAV-SC coverage; ii) the fairness of NC is lower than the one of MC-NES, since the area throughput for this strategy is computed solely from the MC spectral efficiency and MC baseline BW, which strongly vary in our scenario (see Tab. 34); iii) the fairness of BBSR is equal to NC when no UAV-SC is exploited, then lower than NC when (few) UAV-SCs are exploited, and rapidly approaching the fairness of MC-NES when a large number of UAV-SCs is used to cover the areas.

In the following, we consider an additional set of metrics in the range $[0,1]$ to better position BBSR, MC-NES and NC. More in depth, we consider: i) the gain from the energy sold $G^{\mathrm{SELL}}$, ii) the saving on the energy bought $S^{\mathrm{BUY}}$, iii) the fraction of data rate $F^{\mathrm{RATE}}$, and iv) the already introduced fairness index on the rate $J^{\text {RATE }}$. All the metrics are expanded in Tab. 6. Focusing on the expression of $S^{\mathrm{BUY}}$, the denominator $E_{M C-N E S}^{\mathrm{BUY}}$ is the total energy bought by the MC-NES strategy. Focusing instead on the expression of $F^{\text {RATE}}$, the denominator in this case is the total throughput achieved when the UAV-SCs covers all the areas in all the TSs. Fig. 10(d) reports the spider plot with the four considered metrics and the different strategies. The coordinates of the points are given by the values of the metrics in the following order (counter-wise from top):

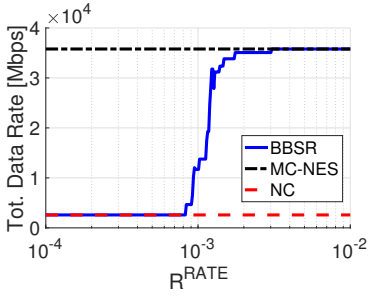

(a) Total data rate $\rho^{\text {TOT }}$

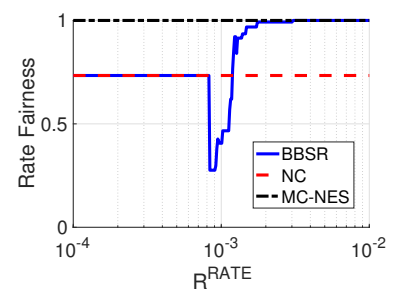

(c) Total data rate fairness $J^{\text {RAT }}$

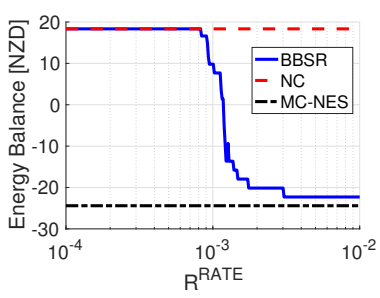

(b) Energy Balance $B^{\text {ENERGY }}$

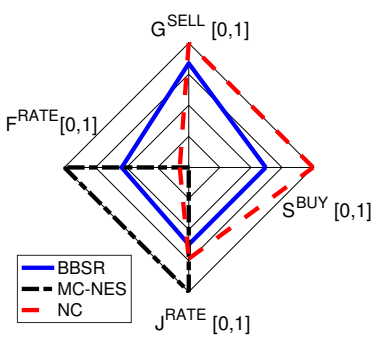

(d) Radar plot $\left(R^{\text {RATE }}=1.2\right.$ $10^{-3} \mathrm{NZD} / \mathrm{Mbps}$ ).
Fig. 10. BBSR, NC and MC-NES comparison $(|T|=144)$.

$\left(0, G^{S E L L}\right),\left(S^{B U Y}, 0\right),\left(0,-J^{R A T E}\right)$ and $\left(-F^{R A T E}, 0\right)$. A line is then used to connect the different points belonging to the same strategy. In addition, we consider the output of BBSR with an intermediate value of the throughput weight $R^{\text {RATE }}=1.2 \cdot 10^{-3}$ [NZD/Mbps]. By analyzing the figure, we can note that NC is oriented in maximizing $G^{S E L L}$ and $S^{\mathrm{BUY}}$, while the values of $F^{\mathrm{RATE}}$ and $J^{\mathrm{RATE}}$ are pretty small. Consequently, the area covered by NC is very unbalanced towards the upper right side of the figure. On the other hand, an opposite trend occurs for MC-NES, which tends to solely maximize $F^{\mathrm{RATE}}$ and $J^{\mathrm{RATE}}$. Interestingly, BBSR is able to wisely balance the different metrics, and presents a pretty wide area almost centered in the figure.

\subsection{BBSR Analysis}

We now evaluate the performance of BBSR in more detail. Fig. 11(a) reports the percentage of served areas (computed across all the areas and all the TSs) vs. the variation of $R^{\mathrm{RATE}}$. Interestingly, BBSR is able to pass from $0 \%$ to $100 \%$ of areas covered by UAV-SCs, depending on the $R^{\text {RATE }}$ values. We then move our attention to the different actions performed by the UAV-SCs (i.e., STAY, MOV, COV and REC). Fig. 11(b) reports the percentage of actions vs. $R^{\mathrm{RATE}}$. When $R^{\mathrm{RATE}}$ is low (left part of the figure), all the UAV-SCs are in the STAY state, i.e., parked at the ground sites and not consuming energy. Then, as soon as $R^{\mathrm{RATE}}$ is increased, the COV, MOV and REC actions start to be applied. At last, for large values of $R^{\text {RATE }}$ (right part of the figure), half of the actions belong to the COV category. This is an expected result, since, with this setting, half of the UAV-SCs are covering the areas, and half are doing other actions. ${ }^{9}$ In the following, we consider the total amount of BW released by the areas to the sites $W^{\mathrm{REL}}$ and the total amount of BW assigned by the sites to the areas $W^{\mathrm{AS}}$. The formal expressions of $W^{\mathrm{REL}}$ and $W^{\mathrm{AS}}$

9. We recall that in our scenario we have set a number of UAV-SCs $|D|=2 \cdot|A|$. 


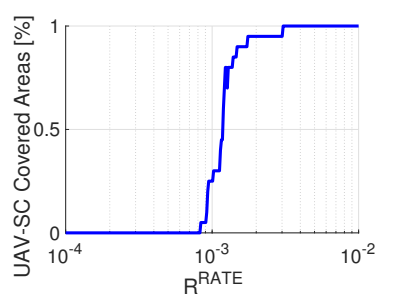

(a) Percentage of UAV-SC covered areas

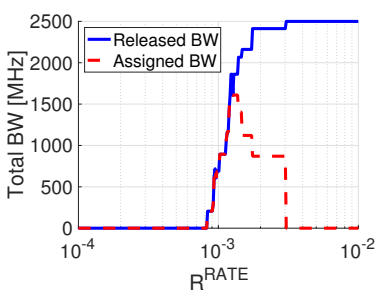

(c) Total BW released/assigned

Fig. 11. UAV-SCs actions, percentage of areas covered by UAV-SCs and total BW released/assigned vs. $R^{\text {RATE }}$ variation for BBSR $(|T|=144)$.

are detailed in Tab. 6, while the values w.r.t. $R^{\mathrm{RATE}}$ variation are shown in Fig. 11(c). Initially, $W^{\mathrm{AS}}=W^{\mathrm{REL}}=0$, since no UAV-SC is used. Then, when $R^{\mathrm{RATE}}$ is increased, the UAVSCs are exploited. This produces a positive $W^{\mathrm{REL}}$, which is entirely consumed by $W^{\mathrm{AS}}$. In other words, all the $\mathrm{BW}$ released by the areas covered by the UAV-SC is used to enhance the BW of the areas not covered by any UAV-SC. This is valid up to a certain threshold of $R^{\mathrm{RATE}}$ (close to 0.001 [NZD/Mbps]), after which $W^{\mathrm{AS}}<W^{\mathrm{REL}}$, i.e., not all the released $\mathrm{BW}$ is assigned to the areas uncovered by UAVSCs. By further investigating this issue, we verify that this condition occurs when: i) a large amount of areas is covered by UAV-SCs (typically more than 50\%), ii) there are sites in which all the corresponding areas are served by UAVSCs. At last, when the UAV-SCs cover all the areas, $W^{\mathrm{REL}}$ is maximized, while $W^{\mathrm{AS}}=0$. However, we point out that, even in this condition, the released BW is not lost, but it is stored in the variables $\gamma_{s}^{t}$. Therefore, this surplus of BW can be redistributed to other part of the territory covered by the MCs (i.e., the extra BW zones reported in Fig. 1).

Finally, we consider the paths that are used by the UAV-SCs when moving from the sites to the covered areas. Fig. 12(a) reports the outcome of BBSR for $R^{\text {RATE }}=$ 0.0012 [NZD/Mbps]. The colored links mark the paths that are activated. In this case, there are paths in which the recharging site and the served area are pretty close (see e.g, the path between site 4 and area 5). However, there are also paths characterized by a longer distance (see e.g, tha path between site 4 and area 10). Moreover, we can observe that most of the areas are not served by any UAVSC. Fig. 12(b) draws the picture when the weight revenue $R^{\text {RATE }}$ is increased to 0.01 [NZD/Mbps]. As expected, all the areas are now served by UAV-SCs. Furthermore, most of the paths are characterized by a long distance between the recharging site and the covered area. A natural question is then: why are longer paths preferred compared to shorter ones? To answer this question, we recall the computation of the horizontal speed $H_{l}$, appearing in Eq. (19). According

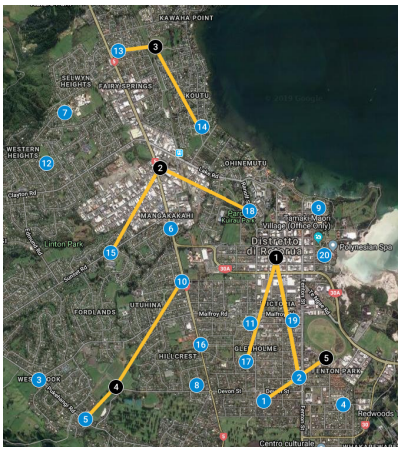

(a) $R^{\mathrm{RATE}}=0.0012 \mathrm{NZD} / \mathrm{Mbps}$

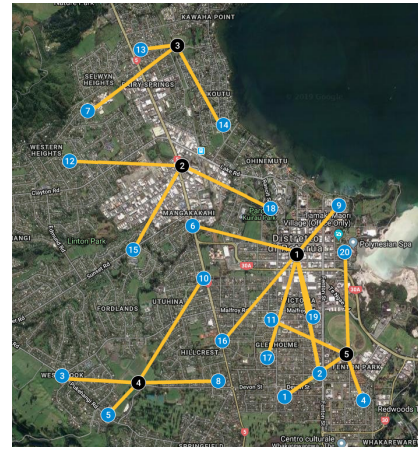

(b) $R^{\text {RATE }}=\cdot 10^{-2} \mathrm{NZD} / \mathrm{Mbps}$
Fig. 12. UAV-SCs paths set by BBSR (Subfigures best viewed in colors).

to Eq. (19), when the distance between a site and an area is increased, $H_{l}$ is increased. This allows to better exploit the lift force, and consequently to decrease the level flight energy consumption of Eq. (18). As a result, the UAVSC consumes less energy when the distance between the recharging site and the serving area is increased.

\section{Conclusions AND Future WORK}

We have focused on the optimization of the energy that is sold to the grid, the energy that is bought from the grid, and the throughput provided to a set of areas in a UAV-aided cellular network. We have modeled the problem by proposing the J-MATE formulation, which is able to adequately take into account all the revenue/cost terms associated to energy and throughput. In addition, we have proposed an efficient heuristic, called BBSR, to solve the problem even for instances composed of hundreds of TSs, which can not be tackled by state-of-the-art optimization tools. Our approaches integrate innovative features, like the redistribution of the MCs BW as a consequence of the UAV-SCs coverage, detailed consumption models for the ground sites and the UAV-SCs, and a multi-period graph approach to control the UAV-SC missions. Results, obtained over a realistic scenario, demonstrate the superiority of JMATE and BBSR w.r.t. the competing solutions. In addition, BBSR is able to reduce both the computation time and the memory occupation of five orders of magnitude compared to J-MATE.

We believe that the presented approach can be extended in a number of directions. First, the possibility to exploit multiple UAV-SCs that simultaneously cooperate to serve the same area is an interesting topic. This step could also integrate a more detailed RRM approach, tailored to single users and different services. Second, the impact of introducing other renewable energy sources (e.g., wind turbines, biogas plants) should be considered. Third, the design of smart algorithms based on machine learning approaches to react to unexpected events/failures should be considered, given the increasingly popularity of UAVs and the demand for communications.

\section{ACKNOWLEDEGMENTS}

This work has received funding from the University of Rome Tor Vergata BRIGHT project (Mission Sustainability 
Call). We thank Vincent Diao, Sam Madanian, and Jing Ma for their help in performing the measurements for the validation of the UAV-SC energy consumption model.

\section{References}

[1] Y. Zeng, R. Zhang, and T. J. Lim, "Wireless communications with unmanned aerial vehicles: opportunities and challenges," IEEE Communications Magazine, vol. 54, no. 5, pp. 36-42, 2016.

[2] M. Mozaffari, W. Saad, M. Bennis, and M. Debbah, "Drone small cells in the clouds: Design, deployment and performance analysis," in 2015 IEEE Global Communications Conference (GLOBECOM), pp. 1-6, IEEE, 2015.

[3] R. I. Bor-Yaliniz, A. El-Keyi, and H. Yanikomeroglu, "Efficient 3$D$ placement of an aerial base station in next generation cellular networks," in IEEE ICC, pp. 1-5, May 2016.

[4] A. Fotouhi, H. Qiang, M. Ding, M. Hassan, L. G. Giordano, A. Garcia-Rodriguez, and J. Yuan, "Survey on UAV cellular communications: Practical aspects, standardization advancements, regulation, and security challenges," IEEE Communications Surveys \& Tutorials, 2019.

[5] M. Mozaffari, W. Saad, M. Bennis, Y.-H. Nam, and M. Debbah, "A tutorial on UAVs for wireless networks: Applications, challenges, and open problems," IEEE Communications Surveys $\mathcal{E}$ Tutorials, 2019.

[6] N. Cheng, W. Xu, W. Shi, Y. Zhou, N. Lu, H. Zhou, and X. Shen, "Air-ground integrated mobile edge networks: Architecture, challenges, and opportunities," IEEE Communications Magazine, vol. 56, no. 8, pp. 26-32, 2018.

[7] V. Sharma, M. Bennis, and R. Kumar, "UAV-assisted heterogeneous networks for capacity enhancement," IEEE Communications Letters, vol. 20, no. 6, pp. 1207-1210, 2016.

[8] L. Chiaraviglio, N. Blefari-Melazzi, W. Liu, J. A. Gutierrez, J. van de Beek, R. Birke, L. Chen, F. Idzikowski, D. Kilper, P. Monti, A. Bagula, and J. Wu, "Bringing 5G into Rural and Low-Income Areas: Is It Feasible?," IEEE Communications Standards Magazine, vol. 1, no. 3, pp. 50-57, 2017.

[9] S. A. R. Naqvi, S. A. Hassan, H. Pervaiz, and Q. Ni, “Drone-aided communication as a key enabler for $5 \mathrm{G}$ and resilient public safety networks," IEEE Communications Magazine, vol. 56, no. 1, pp. 3642, 2018.

[10] S. Sekander, H. Tabassum, and E. Hossain, "Multi-tier drone architecture for 5G/B5G cellular networks: Challenges, trends, and prospects," IEEE Communications Magazine, vol. 56, no. 3, pp. $96-$ $103,2018$.

[11] Ø. Magnussen, G. Hovland, and M. Ottestad, "Multicopter UAV design optimization," in 2014 IEEE/ASME 10th International Conference on Mechatronic and Embedded Systems and Applications (MESA), pp. 1-6, IEEE, 2014.

[12] L. Amorosi, L. Chiaraviglio, F. D'Andreagiovanni, and N. BlefariMelazzi, "Energy-efficient mission planning of UAVs for 5G coverage in rural zones," in IEEE International Conference on Environmental Engineering (EE), pp. 1-9, 2018.

[13] M. Mozaffari, W. Saad, M. Bennis, and M. Debbah, "Efficient deployment of multiple unmanned aerial vehicles for optimal wireless coverage," IEEE Communications Letters, vol. 20, no. 8, pp. 1647-1650, 2016.

[14] H. N. Qureshi and A. Imran, "On the tradeoffs between coverage radius, altitude and beamwidth for practical uav deployments," IEEE Transactions on Aerospace and Electronic Systems, 2019.

[15] S. Mignardi and R. Verdone, "On the performance improvement of a cellular network supported by an unmanned aerial base station," in 2017 29th International Teletraffic Congress (ITC 29), vol. 2, pp. 712, IEEE, 2017.

[16] S. Jeong, O. Simeone, and J. Kang, "Mobile edge computing via a uav-mounted cloudlet: Optimization of bit allocation and path planning," IEEE Transactions on Vehicular Technology, vol. 67, no. 3, pp. 2049-2063, 2018.

[17] R. Verdone and S. Mignardi, "Joint Aerial-Terrestrial Resource Management in UAV-Aided Mobile Radio Networks," IEEE Network, vol. 32, no. 5, pp. 70-75, 2018.

[18] Q. Wu, Y. Zeng, and R. Zhang, "Joint trajectory and communication design for multi-UAV enabled wireless networks," IEEE Transactions on Wireless Communications, vol. 17, no. 3, pp. 21092121, 2018.
[19] K. Zhu, X. Xu, and S. Han, "Energy-Efficient UAV Trajectory Planning for Data Collection and Computation in mMTC Networks," in 2018 IEEE Globecom Workshops (GC Wkshps), pp. 1-6, Dec 2018.

[20] R. Li, Z. Wei, L. Yang, D. W. Kwan Ng, N. Yang, J. Yuan, and J. An, "Joint Trajectory and Resource Allocation Design for UAV Communication Systems," in 2018 IEEE Globecom Workshops (GC Wkshps), pp. 1-6, Dec 2018

[21] A. Mardani, M. Chiaberge, and P. Giaccone, "CommunicationAware UAV Path Planning," in 2018 6th IEEE International Conference on Wireless for Space and Extreme Environments (WiSEE), pp. 12 17, Dec 2018.

[22] Y. Zeng, J. Lyu, and R. Zhang, "Cellular-connected UAV: Potential, challenges and promising technologies," IEEE Wireless Communications, 2018.

[23] A. Trotta, M. D. Felice, F. Montori, K. R. Chowdhury, and L. Bononi, "Joint Coverage, Connectivity, and Charging Strategies for Distributed UAV Networks," IEEE Trans. on Robotics, vol. 34, pp. 883-900, Aug 2018.

[24] Q. Wu, L. Liu, and R. Zhang, "Fundamental Trade-offs in Communication and Trajectory Design for UAV-Enabled Wireless Network," IEEE Wireless Communications, vol. 26, no. 1, pp. 36-44, 2019.

[25] M. Hua, Y. Wang, C. Li, Y. Huang, and L. Yang, "Energy-Efficient Optimization for UAV-aided Cellular Offloading," IEEE Wireless Communications Letters, 2019.

[26] Y. Sun, D. Xu, D. W. K. Ng, L. Dai, and R. Schober, “Optimal 3Dtrajectory design and resource allocation for solar-powered UAV communication systems," IEEE Transactions on Communications, 2019.

[27] L. Chiaraviglio, F. D'Andreagiovanni, K.-K. R. Choo, F. Cuomo, and S. Colonnese, "Joint Optimization of Area Throughput and Grid-connected Microgeneration in UAV-based Mobile Networks," IEEE Access, 2019.

[28] M. R. Garey and D. S. Johnson, "Computers and intractability: A guide to the theory of np-completeness," 1979.

[29] "Cellmapper." https://www.cellmapper.net. Last Accessed: 201907-26.

[30] C. Ide, R. Falkenberg, D. Kaulbars, and C. Wietfeld, "Empirical analysis of the impact of lte downlink channel indicators on the uplink connectivity," in 2016 IEEE 83rd Vehicular Technology Conference (VTC Spring), pp. 1-5, IEEE, 2016.

[31] "Small cell networks and the evolution of 5g." https://www.qorvo.com/design-hub/blog/ small-cell-networks-and-the-evolution-of-5. Last Accessed: 2019-08-07.

[32] S. Colonnese, F. Cuomo, L. Chiaraviglio, V. Salvatore, T. Melodia, and I. Rubin, "CLEVER: a cooperative and cross-layer approach to video streaming in hetnets," IEEE Transactions on Mobile Computing, vol. 17, no. 7, pp. 1497-1510, 2018.

[33] B. H. Jung, H. Leem, and D. K. Sung, "Modeling of power consumption for macro-, micro-, and RRH-based base station architectures," in 2014 IEEE 79th Vehicular Technology Conference (VTC Spring), pp. 1-5, IEEE, 2014.

[34] B. Marion and M. Anderberg, "PVWATTS - an online performance calculator for grid-connected PV systems," in Proc. of the solar conference, pp. 119-124, American Solar Energy Society, 2000.

[35] "Domestic generation." https://www.trustpower. co.nz/our-assets-and-capability / power-generation/ domestic-generation. Last Accessed: 2019-07-23.

[36] "NZ Electricity Authority Wholesale information and trading system (WITS)." https://www.ea.govt.nz/operations/wholesale/ spot-pricing/wits/. Last Accessed: 2018-12-21. 